\title{
DEGASSING AND PURIFICATION OF BOILER FEED-WATER.
}

By PaUl KESTNER, OF Paris.

How to feed boilers with water and to enable them to run for long periods under -steam without scale or corrosion is the problem which becomes more urgent every day, and further, it is a problem on which a great economy of fuel and material depends. It is certain that a large number of technical men have already attacked the problem-frequently with considerable success, but never with complete success. The old lime and soda purifying apparatus, of very large dimensions, has been gradually replaced by apparatus of a much smaller size in which, thanks to the arrangements which will be discussed, the rate at which purification is effected has been increased. Lately, purification has been done by heating, which has accelerated the process. In spite of these efforts, some kinds of water have proved refractory to treatment; after addition of lime and soda, when raised to high temperatures, they have still given rise to scale in the boilers. A process which has been effective with one kind of water has given only indifferent results when used with another water. Another process which has eliminated all calcareous salts from the water has, in some boilers produced a concentrated solution of soda. Perfect purification is rarely obtained.

There is, however, a process which ensures the prevention of the formation of mud and incrustation in boilers. This process, тा 2 
which is that of continuous blowing-down, will be considered in this Paper. It is necessarily used in conjunction with the hot purification process, and the combination constitutes a complete method of treating the boiler feed-water.

Purification at High Temperature.--It is unnecessary to recall the advantages of feeding boilers with hot water. In addition to those advantages due to the thermal side of the question, there

Fra. 1.

Curves showing Reduction in Time required for the same Reactions in Hot and Cold Liquids.

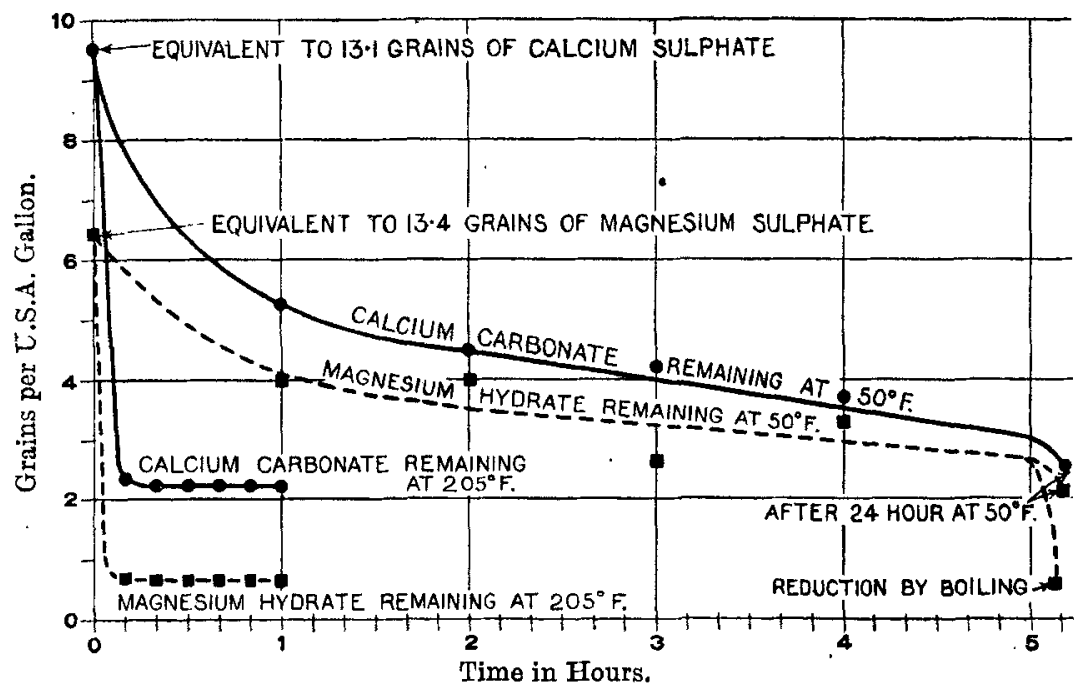

are those produced by the reduced solubility of gases in water at high temperatures, as will be shown. Purification at ordinary temperatures, whether by the use of soda alone or lime and soda, requires considerable time, owing to the slowness of the reaction and the fineness of the precipitate formed, which settles very slowly. The reduction in the time required for the same reactions in hot and cold liquid is shown in the curves, Fig. 1, which has been taken from a catalogue published by Messrs. Cochran and Cọ. 
Ingenious processes have been invented for enabling the speed of these reactions to be increased and to hasten precipitation in cold water. This consists in bubbling through the sediment in the water to be purified after the addition of the reagents. Under the influence of the large quantity of old residues, the size of the particles that are formed increases; the small particles that come into existence are attracted by the larger ones that have already formed which absorb them, and this precipitate, which consists of much coarser material, settles very much more rapidly. Owing to the increased speed of settling, the reagent quickly becomes poor in one of its constituents, and the equilibrium of the reaction is favourably disturbed. This process allows the rate of purification to be increased, and hence the dimensions of the apparatus can be reduced; but, on the other hand, a difficulty is generally introduced where agitation of the sediment is obtained by a stream of water which carries air with it. This causes a considerable increase in the amount of oxygen contained in the water that is to be purified; under the conditions met with in practice saturation occurs almost instantaneously and gives rise to corrosion in boilers.

In any process for softening water, a rise of temperature gives the following advantages:-

(1) Increase of the Speed of Reaction.-For the same initial concentration, equilibrium is obtained, as is well known, more rapidly at higher temperatures than at the temperature of the air. This follows from the laws of Guldberg and Wage, Gibbs and Le Chatelier, to which there is no reason to refer further. We need only mention that at the temperature of absolute zero, i.e.,- $273^{\circ} \mathrm{C}$. reactions have no speed at all, and that any rise of temperature increases the speed of obtaining equilibrium in any reaction. In the case of the reactions with which we are concerned, and which are of the character of double decomposition, equilibrium is only slightly affected by rise of temperature, but the speed at which it is obtained is enormously increased.

(2) Diminution of the Viscosity of the Water.-The coefficients of viscosity decrease very rapidly as is shown by the following curve, Fig. 2 (page 294). 
Viscosity is the chief resistance which is opposed on the one hand to molecular attraction of the particles of the precipitate towards each other in the formation of larger aggregations, and on the other hand to the settlement of the particles formed from suspension in the solution in which the precipitate has been produced till they reach the bottom of the containing vessel. The coefficient

FIa. 2.-Curves showing Rapid_Decrease of the Coefficients of Viscosity.

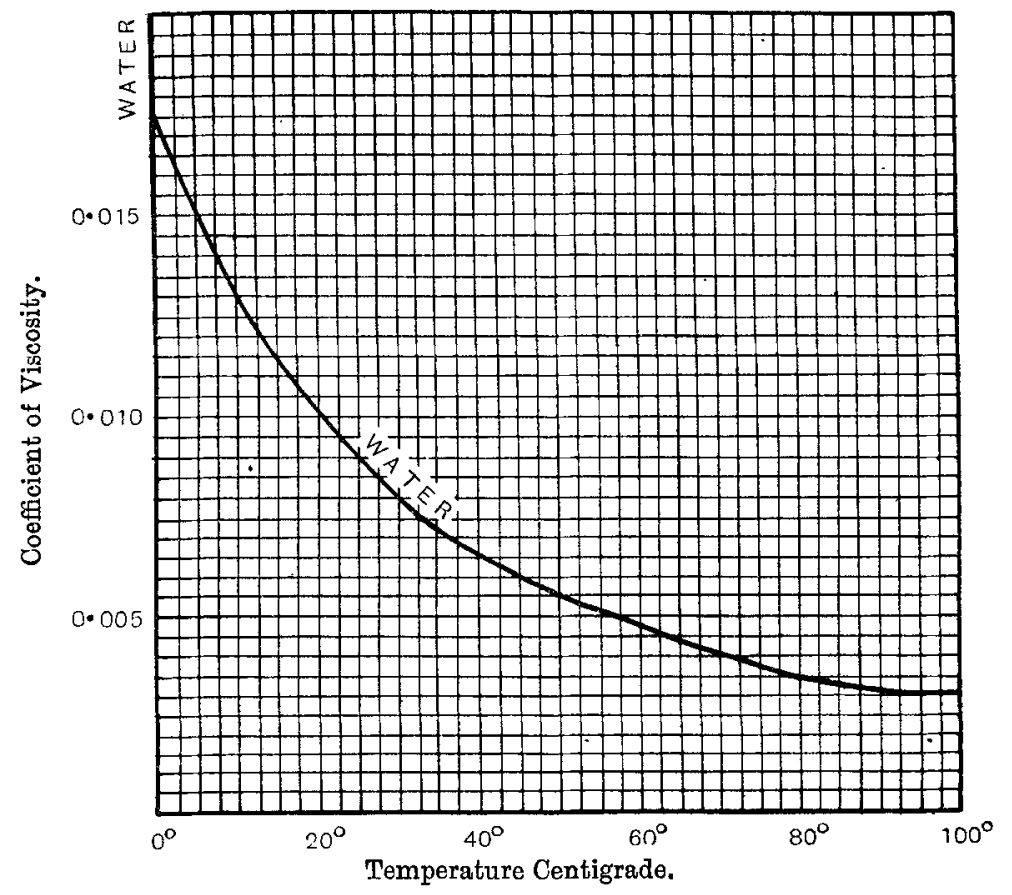

of viscosity is the tangential force required to displace an unit area at unit velocity in the medium under consideration. It will be seen, therefore, that the rate of forming a granular precipitate depending on molecular attraction will, other things being equal, be directly proportional to the coefficient of viscosity. The velocity of falling of a spherical particle having a density greater than water will be given by Stokes's formula. 


$$
\mathrm{V}=\frac{2}{9} \cdot \frac{\mathrm{D}-d}{\mathrm{~N}} \cdot g \cdot r^{2}
$$

in which $\mathrm{V}$ is the velocity, $\mathrm{D}$ the density of the particle, $d$ the density of the water, $g$ the acceleration due to gravity, $r$ the radius of the particle, and $\mathrm{N}$ the coefficient of absolute viscosity.

If we examine the curve of coefficients of viscosity, we shall find that this maximum velocity of falling will, under some conditions, be four times as great at $100^{\circ} \mathrm{C}$. as it is at $12.5^{\circ} \mathrm{C}$. We may therefore deduce that the deposition of a sediment will be four times more rapid with water at boiling point, and that the volume of the purifier may be reduced in the same proportion.

(3) Decomposition of the Bicarbonates of the Alkaline Earths.-The direct effect of temperature on bicarbonates of the alkaline earths is to decompose them at $70^{\circ} \mathrm{C}$. into carbonic acid gas and the carbonates of the alkaline earths, without the addition of any reagent.

(4) The Diminution in the Solubility of Sulphate of Calcium.Sulphate of calcium becomes reduced at higher temperatures and the precipitation of part of this salt is effected without the intervention of chemical reagents. This reduction is of very slight importance in the process using continuous blow-down, just as the increase of solubility of calcium carbonate is a negligible factor. We shall see later why these facts have so small an influence in the particular case under consideration.

Continuous Blow-down.-When a boiler, the feed-water for which is purified by any method, has been running for some time, all the salts carried in with the feed-water are concentrated in it. These salts are only alkaline salts when the water has been thoroughly purified, and the calcareous sediment of the water has been incompletely purified. In general, a definite amount of calcium carbonate always remains in solution in the water. When the concentration produced by evaporation becomes so high that, notwithstanding the rise of temperature, this carbonate remains insoluble, it is then deposited on the walls of the boiler and in the tubes forming incrustation, which is the harder the more slowly the 
deposit is made. In boilers generating steam very rapidly, these deposits are formed more rapidly than in slow evaporation boilers, and notwithstanding the violent circulation of the water, sediment may accumulate in the tubes. These sediments give rise to overheating and to very hard incrustation.

To avoid these difficulties, in actual practice, the boiler is blown off, that is, at the lowest point on the boiler shell, the water containing the collected mud is drawn off and turned into the drain. This water is at the pressure and temperature of the boiler, and, in blowing off, a large quantity of heat is lost. To be effective, blowing off must take place to the extent of a large actual quantity of water, though the amount is small in proportion to the total contents of the boiler. Continuous blow-down allows these calories to be saved. Instead of sending the blow-off direct into the drain a relatively small quantity is caused to leave the boiler continuously from its lowest point. This water cools in giving up its calories to the feed-water in a feed-water heater of ordinary pattern; the mud is deposited in a trap; it loses in cooling a certain proportion of the salts which had been retained in solution owing to the high temperature of the liquid. Then, the salts which would cause incrustation, mud and deposit having been collected and removed, the liquid is mixed with the feed-water and returns into the boiler. It will thus be seen that there is no loss of calories due to the blowoff, and that neither deposition nor scale can be caused in the boiler. There is, however, another cleansing operation to perform. It is the removal of the mud deposit at the blow-off outside the boiler. This removal is only of small magnitude, because the apparatus in which precipitation takes place has a conical bottom suited to this purpose; and, further, the water in it is not under pressure; it removes only a few calories because the blow-off water is at this moment already considerably cooled. This small amount of extraction at low temperature completely suffices to limit the concentration of the soluble salts before danger from the raising of the boiling point of the water commences.

If the boiler has been blown down to the extent of 20 per cent of its contents in an hour, this means that its contents are renewed 
every five hours. This is impossible to effect by any other process, and it ensures the elimination of all mud that has been produced before it has had time to accumulate. This shows the great advantage presented by the process of continuous blowing-down in itself, this advantage being twofold when the feed-water is purified by means of soda; in fact, in this case, the calcareous and magnesium bicarbonates contained in the water give, by double decomposition with sodium carbonate, sodium bicarbonate and calcareous carbonates according to the reaction :-

$$
\left(\mathrm{CO}_{3}\right)_{2} \mathrm{Ca} \mathrm{H}_{2}+\mathrm{CO}_{3} \mathrm{Na}_{2}=2 \mathrm{CO}_{3} \mathrm{Na} \mathrm{H}+\mathrm{CO}_{3} \mathrm{Ca} \text {. }
$$

The bicarbonate, which is unstable at the temperature at which the purification in hot water takes place, decomposes, at any rate partially, giving a neutral carbonate and carbonic acid gas, according to the equation

$$
2 \mathrm{CO}_{3} \mathrm{NaH}=\mathrm{CO}_{2}+\mathrm{H}_{2} \mathrm{O}+\mathrm{CO}_{3} \mathrm{Na}_{2} .
$$

The water which enters the boiler contains, therefore, carbonate and bicarbonate of soda. These salts are in quantities equivalent to the calcareous salts that have been eliminated. In the boiler the bicarbonate is decomposed, giving a carbonate, as has been seen above, and then the carbonate in its turn is dissociated in the presence of water, giving caustic soda. There is thus another reversible condition of equilibrium in functions of the initial concentration on the one hand and of the temperature on the other. This may be represented in the following form :-

$$
\mathrm{CO}_{3} \mathrm{Na}_{2}+\mathrm{H}_{2} \mathrm{O}=2 \mathrm{NaOH}+\mathrm{CO}_{2} \text {. }
$$

Thus hydrolysis gives more caustic soda, the higher the te mperature.

With continuous blowing-down, water containing caustic soda and carbonate of soda is therefore drawn from the boiler. If this solution be mixed with untreated water, the caustic soda and the carbonate they contain will purify the water; the carbonate in the same manner as previously mentioned, and the caustic soda in the following manner :-

$$
\left(\mathrm{CO}_{3}\right)_{2} \mathrm{Ca} \mathrm{H}_{2}+2 \mathrm{NaOH}=\mathrm{CO}_{3} \mathrm{Na}_{2}+2 \mathrm{H}_{2} \mathrm{O}+\mathrm{CO}_{3} \mathrm{Ca} \text {. }
$$


The caustic soda reacts on the calcium bicarbonate as would calcium oxide, but much more rapidly. New products will then be formed, namely, sodium carbonate and bicarbonate, which will be returned to the boiler.

If the quantity of blowing-down is calculated in such a manner that it brings down the quantity of soda necessary in a given time for the purification of the water entering the boiler in the same time, it will be seen that it will not be necessary to add any carbonate of soda for purification of the temporary hardness of the water. A closed circuit will be formed containing a certain quantity of soda, which will never be exceeded. The soda is never concentrated in the boiler, because no fresh soda is introduced. The concentration in the boiler depends on the proportion of blow-down in relation to the feed-water. The less the quantity of blow-down, the greater will the concentration become, so that the same quantity of salt will be contained in a smaller volume.

It is therefore of the greatest importance to combine purification by soda with the continuous blow-down. This allows of more economical use of the chemical reagents necessary for freeing the water from the bicarbonates of the alkaline earths. Consequently, owing to the circulation of the blow-down through the purifier, there is a blowing through the deposit favourable to drawing-off and purification at high temperature by using the calories that would be thrown away in the drain under ordinary methods of blowing-off. The concentration of the soda in the boiler is limited, and neither deposit nor incrustation can be produced. The consumption of soda is reduced to that necessary for the decomposition of noncarbonates. The Author has devised the following apparatus using a blow-down on the preceding principle, Fig. 3.

This apparatus consists of a steam separator $S$ into which the blow-down enters in the form of a mixture of steam and water. The liquid from the blow-down falls into a central reservoir $P$, in which it gives up its heat to the water surrounding it in the annular chamber $B$, into which it is decanted.

The steam from the blow-down rises in the reheater $R$, in which it reheats the feed-water, which loses a part of its bicarbonate 
FIG. 3.-Blow-down Apparatus enabling the Thermal and Chemical Properties to be saved. (Kestner.)
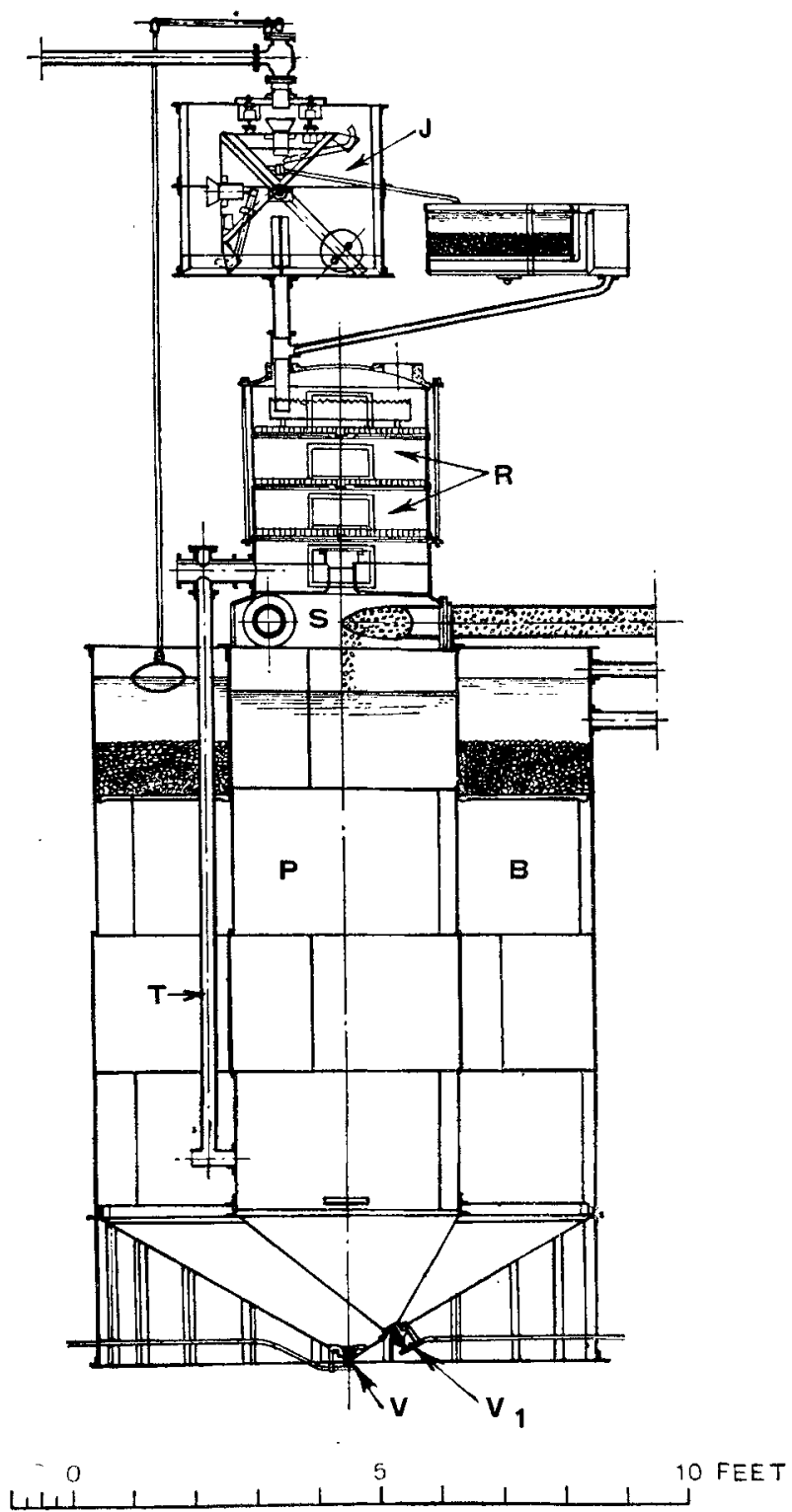
owing to the rise of temperature. The water thus heated falls through the tube $\mathrm{T}$ and mixes with the blow-down as it leaves the central chamber, and then rises in the annular chamber $B$, in which precipitation and settlement take place. Reheated by admixture with the blow-down, as well as by exchange of heat with the central vessel, the purified water passes through the filter and enters the boiler. The soda is distributed in the apparatus by means of a water-meter $J$, which admits it in proportion to the new water (make-up feed). The valves $V$ and $V_{1}$ allow the mud to be separated. This apparatus enables the thermal and chemical properties of the blow-down to be utilized, or, in other words, heat and chemicals are saved.

Position of the Blow-down.-It is necessary to arrange the blowdown at the lowest possible level on the boiler without risk of emptying it. A simple arrangement is recommended by which, when the water of the boiler falls below a certain level, the siphon action of the blow-off is destroyed. At this moment steam leaves by the inlet and calls attention by sounding a whistle. In any case it is impossible to empty the boiler by the continuous blow-down, Fig. 4.

On the pipe of the continuous blow-down and the outlet from the boiler, a reducing valve is placed, which reduces the pressure of the blow-down from that of the boiler to a pressure of between one and two kilogrammes per square centimetre ( 15 to $30 \mathrm{lb}$. per sq. in.) before it enters the purifier. In order to allow of the amount of the blow-down being regular, a meter is placed at the outlet of the boiler and is fitted with a regulating valve, Figs. 5 and 6.

Proportions of the Blow-down.-The proportions of the blow-down are calculated according to the temperature to be maintained in the purifier. It is always desirable that the temperature at which purification is effected should not be less than $70^{\circ} \mathrm{C}$. According to the working pressure of the boiler, various rates of blow-down may be used, and these can be obtained by the eurves, Fig. 7 (page 302). 
FIa. 4.-Contimuous Blow-down.

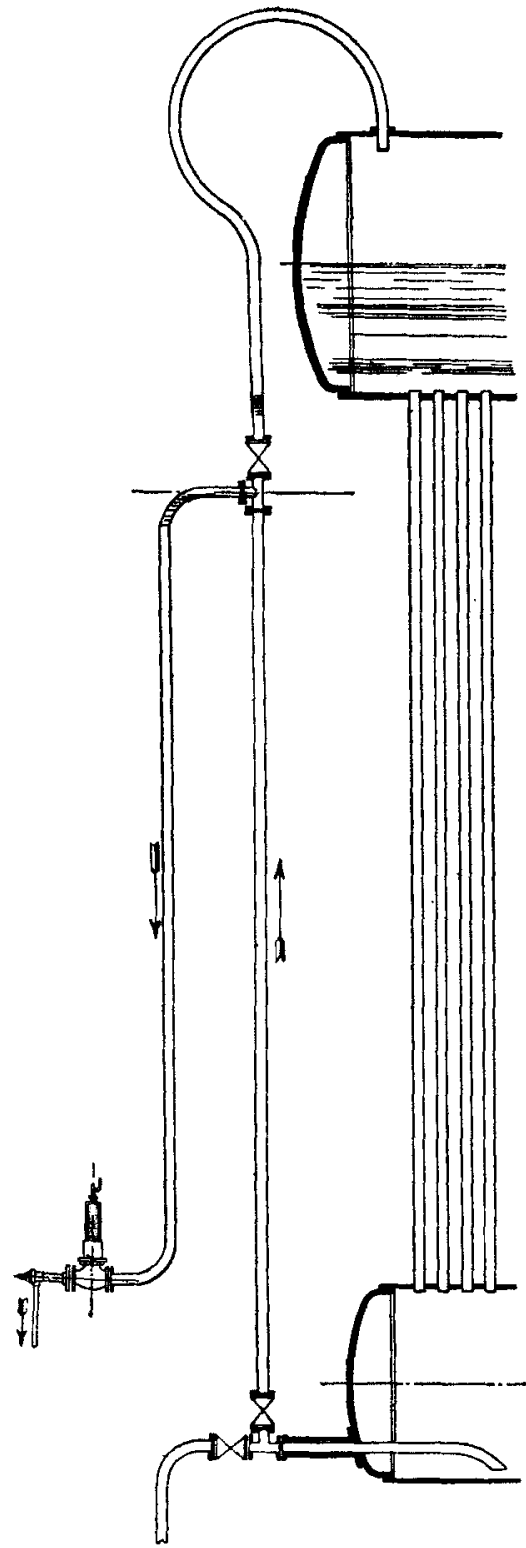

- . EIG. 5.-Regulating Valve.

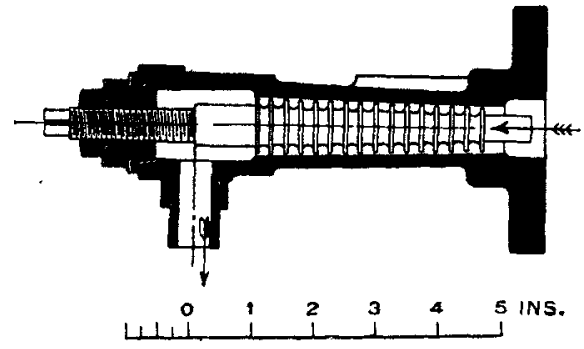

Fia. 6.- F'low Indicator.

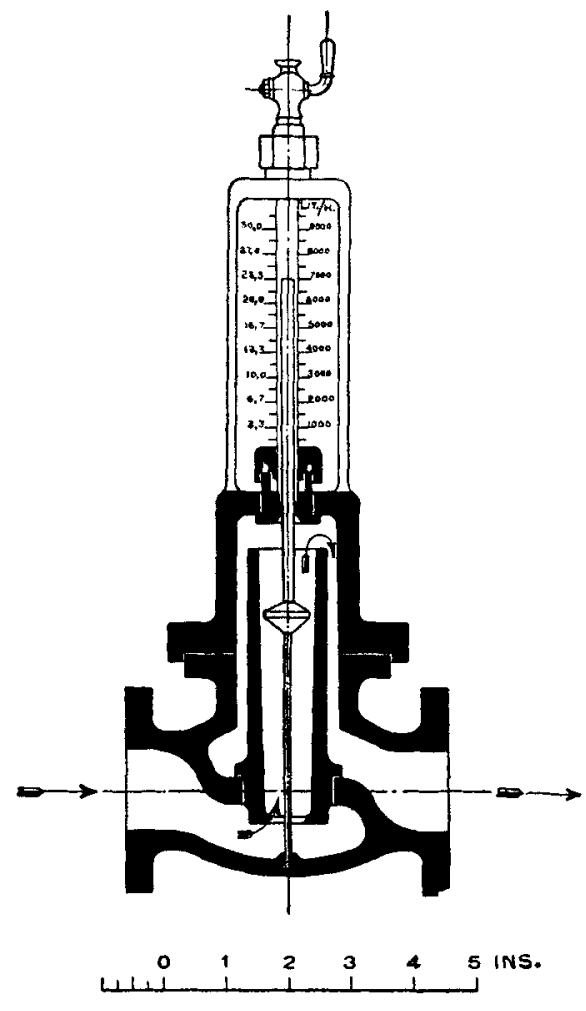


The temperature at which the water leaves the purifier, although very nearly $100^{\circ} \mathrm{C}$., allows of the use of an economiser, as has been shown by recent researches in France.

Central Stations using Distilled Water.-It has just been shown how the system of continuous blow-down can be applied to a boiler

FIG. 7.-Curve showing Temperature of Water leaving Heater Settler. (Water Purification.)

Pressure in lb. per sq. inch in Boiler

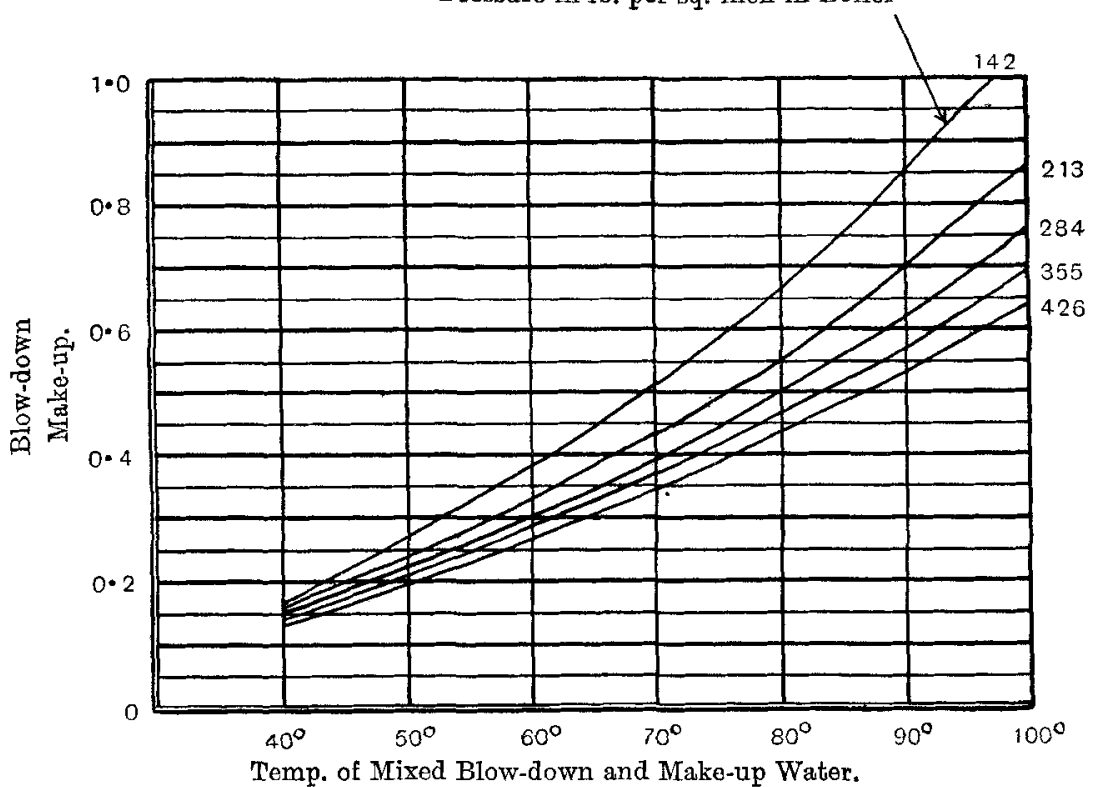

using purified water. A case offering still greater interest is that of central stations using distilled water.

The distilled water of industrial appliances differs greatly from the distilled water of the laboratory. In these appliances, it is necessary to blow down the boilers as a concentration of the boiler contents arises, due to the entry of salts through accidental leakages of impure water in the condensating plant; thus the water that has been distilled contains numerous soluble salts, present in 
smaller proportions than in purified water, but by no means negligible. The following relationship is found to exist between the water in the boiler and the water of distillation:-

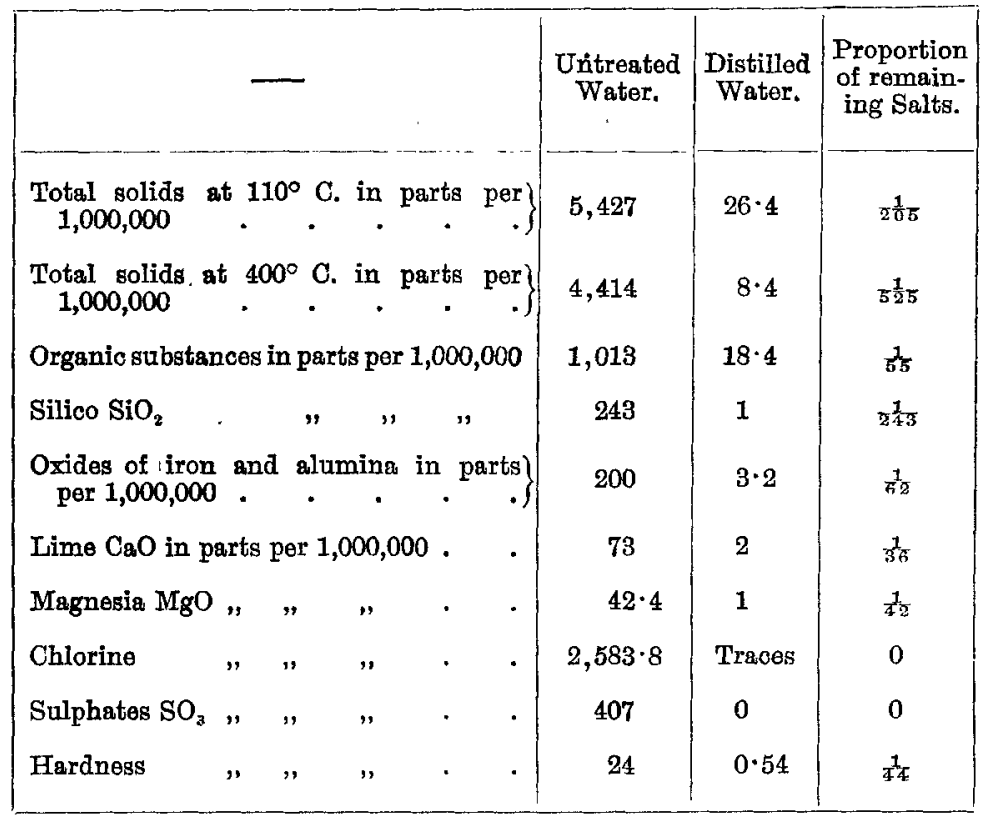

In boilers using distilled water, this water in time becomes a fairly concentrated liquid. Organic matter-oils and grease particularly-is present in considerable quantity, and of these only a small quantity is necessary to produce priming. It is, therefore, necessary to avoid this concentration, and continuous blowing-down is consequently applicable in this case also.

Boilers working with distilled water therefore require to be blown-down occasionally, but, owing to the small amount of salt that they contain, it is useless to remove a large quantity of liquid. These boilers are consequently blown-down to the extent of 1 or 2 per cent only, which means that the quantity of saline solution is replaced in the boiler by distilled water. By this method the increase in the quantity of dissolved matter is necessarily limited. 
It could not, for example, exceed fifty times the concentration of the distilled water taken from the condenser, in the case of a boiler blown down to the extent of 2 per cent. In the case quoted above, this corresponds to a dry residue of $1 \cdot 3 \mathrm{grm}$. per litre, which is certainly not negligible. The water blown down is of no special interest so far as its chemical composition is concerned; but it is hot water which enters the distilling apparatus without loss of its calories.

Even when an evaporator is used, the continuous blowing-down method can be adopted. A certain amount of saline or impure water can be drawn from the boilers and passed into the evaporator; this latter returns it to the boiler after it has been freed of the greater portion of its salts. It will be seen later how the simultaneous use of continuous blowing-down, in conjunction with degassing, allows of the establishment of a complete closed water circuit in a central station using distilled water.

Experience has shown that boilers fed with distilled water as well as those fed with purified water are liable to deterioration due to corrosion of the plates. The interior of the boiler, which is smooth and clean when put to work, becomes covered in places with a thin coating of rust; the small patch of rust increases in size little by little; the rust forms a scale of somewhat spongy nature, and when the boiler is put off work, or frequently even while it is still at work, for various reasons this scale ceases to adhere to the plate and falls from it. In its place there is formed a slight depression, caused by the disappearance of the metal which has entered into combination in forming rust; the depression becomes again rusty, and eats further into the plate by repetition of the process. Some boilers, after having been a certain time at work, have their plates pitted all over so that they recall the battered and dented walls of a village that has been under fire during the war. The strength of the boiler is undermined, and it becomes necessary to renew the damaged plates, or even the whole boiler.

Although the commencement of corrosion does not always become visible, it is nevertheless the cause of serious damage to boilers and shortens their life. Owing to the high first cost of a boiler, an important economy is effected by extending its working 
life by one or more years. This can be done by preventing corrosion.

The Causes of Corrosion.-What is the origin of corrosion? In the case of boilers using distilled water there can be no doubt. Oxidization of the plate can only be produced by oxygen dissolved in the feed-water and set free in the boiler. When the rate of evaporation is steady and the boiler is in regular work, the dissolved air is released at those portions of the boiler in which the water has not reached the boiling point, or where the circulation is sluggish. At these places bubbles are formed which cling to the surface, and leave their oxygen in combination in the form of rust. There is also the freeing of air-bubbles at the water level when the rate of evaporation is reduced. It is well known that intermittent working is the most destructive cause of all.

The combination that takes place between the oxygen and the iron can be explained in several ways, as may be seen by reference to the work of Newton Friend.* The usually accepted theory is that oxygen, in the presence of water vapour, combines directly with iron according to the following reaction :-

$$
2 \mathrm{Fe}+3 \mathrm{H}_{2} \mathrm{O}+3 \mathrm{O}=2 \mathrm{Fe}(\mathrm{OH})_{3} \text {. }
$$

Treadwell's theory, according to which carbonic gas acts as catalyzer, is also interesting, and leads to the same result :-

$$
\begin{gathered}
\mathrm{Fe}+\mathrm{CO}_{2}+\mathrm{H}_{2} \mathrm{O}=\mathrm{CO}_{3} \mathrm{Fe}+\mathrm{H}_{2} \\
2 \mathrm{CO}_{3} \mathrm{Fe}+3 \mathrm{H}_{2} \mathrm{O}+\mathrm{O}=2 \mathrm{Fe}(\mathrm{HO})_{3}+2 \mathrm{CO}_{2} .
\end{gathered}
$$

It is, however, to be noted that even when there is no carbonic acid gas, so far as our methods permit its presence to be detected, rusting occurs. This is disputed by some authorities, but whatever the actual process, it cannot be denied that oxygen is the cause of rust. Numerous electrical theories have been proposed to explain the oxidization of iron. These are not, however, applicable to the case of boilers using distilled water, where electrolytic action is

"The Corrosion of Steel and Iron." 
minute and the weakness of the saline solution in the boilers is unfavourable to the production of local currents.

All these theories, taken individually, appear very plausible

FIG. 8.-Relation between Corrosive Power and Concentration for a Number of Solutions.

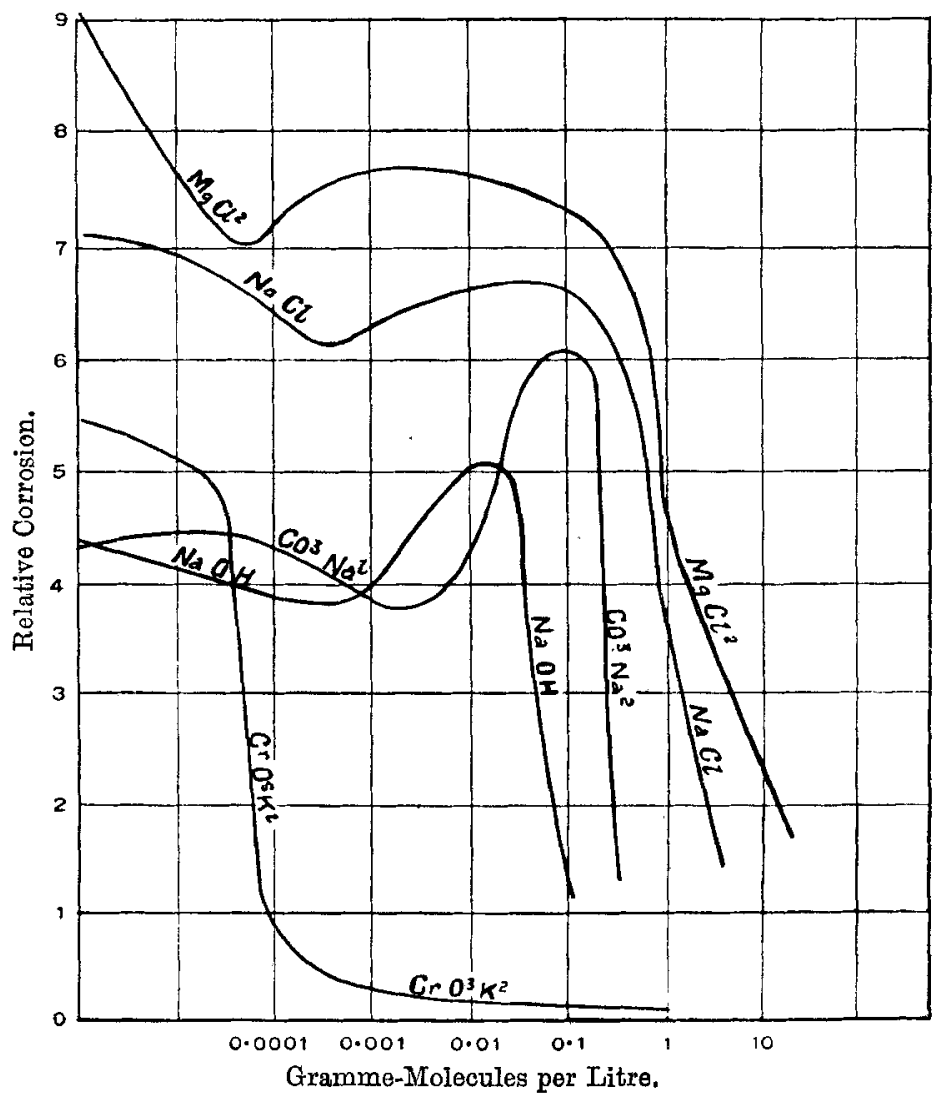

and might form the subject of a lengthy chemical investigation, into which it is not proposed to enter, as it is of academic interest only.

If the action of oxygen is obvious and well known in boilers using distilled water, it is not so clear in the case of boilers using 
purified water, or it may be considered less important on the ground that the concentration of salts, obtained under this system of working, forms the principal factor in the eating away of the plates. This erroneous view depends generally on an incomplete appreciation of the facts; it has, moreover, been proved that although highly concentrated solutions attack the gun-metal fittings more severely, their action on iron is greatly reduced.

If we examine the corrosive effect of saline solutions, we find that in general it begins by increasing in proportion to the strength of concentration up to a critical point at which it attains a maximum ; the effect then decreases rapidly as the concentration proceeds. The diagram, Fig. 8, shows the relation between corrosive power and concentration for a number of solutions. From these curves it appears that there is a certain critical concentration which must be exceeded in working boilers. In practice, after a few hours' work, a boiler using purified water will have already attained a concentration fully one hundred times the critical amount. Concentrated solutions may, therefore, give rise to a higher boiling point, to priming, and to the deposition of crystals, but corrosion must not be included in the list.

Electrical action may have some influence on the preceding. In fact stray currents may find suitable conductivity in the salt solutions, but boilers are not made of a single piece of plate, and the interruptions caused by the joints tend to localize the effects of the current. Moreover, it appears probable that if currents of sufficiently high voltage existed, they would soon cause serious damage, the only remedy for which would be the adoption of insulation of the boiler. Generally the boiler seatings are very dry and offer no facility for the passage of electric currents. It may happen that galvanic pairs are formed between parts of the boiler which differ in chemical composition or physical nature, but these pairs are generally of but feeble strength and their electrolytic effect would be slight and confined to small areas.

There is still the question of electrolytic dissociation. This occurs much more easily in a dilute than in a concentrated solution, which confirms the previous statement; in general it is of much 
less importance than the phenomena to which corrosion is attributed.

Hydrolysis may have much more important effects. A particularly important case, and the best known, is that of magnesium chloride, which gives rise to the production of hydrochloric acid, and is liable to cause indefinite corrosion. Magnesium chloride in the presence of water may be decomposed into magnesia and hydrochloric acid which attacks the plates vigorously.

$$
\mathrm{MgCl}_{2}+\mathrm{H}_{2} \mathrm{O}=\mathrm{MgO}+2 \mathrm{HCl} \text {. }
$$

Fortunately this salt only occurs occasionally, and is sufficiently well known for special care to be taken where the feed-water contains magnesium chloride. Acids attack the plates, but, apart from the case just mentioned, the only acid found in boilers is the carbonio acid introduced in the feed-water. Carbonic acid alone has only feeble action on iron; in the laboratory a cold concentrated solution of the gas produces only a few bubbles of hydrogen on a piece of iron immersed in the solution. If, however, oxygen is present, the metal is attacked much more rapidly, because the ferrous carbonate formed decomposes into rust and carbonic acid which reommences the action. In this case also it is oxygen that produces the corrosion.

Oxygen comes into play still further when the water contains salts capable of combining with oxygen and forming unstable oxygen-compounds which can part with their oxygen to form other oxides. Among these salts some, particularly certain oxychlorides, are known and have been separated; but the hypothesis, that certain oxy-salts that have not yet been separated may be present, affords an explanation of the rapid eating away of iron by sodium chloride in the presence of air, whereas solutions of the same strength, absolutely free from oxygen, can be kept in contact with the metal without effecting any change whatever.

Thus it appears that it is the solution of atmospheric oxygen in water that is the chief cause of corrosion, whether the feed is distilled water or purified water. This is supported by the fact that corrosion is often more serious in boilers using distilled water, 
in which dissolved oxygen can be the only agent, than in boilers using purified water.

The remedy therefore appears to be very simple, namely, the elimination of the dissolved oxygen.

Oxygen in Solution in the Feed. - Atmospheric air (and therefore the oxygen that it contains) dissolves in water almost instantaneously up to the point of saturation for the particular temperature and pressure. The solubility of air in water is proportional to the pressure, and inversely proportional to the temperature ; it follows Henry's law which, though approximate, is very nearly true for the case of air mixed with a gas that is not very soluble in water. Each of these gases dissolves in proportion to its share in the pressure, as though alone; thus air contains four volumes of nitrogen to one volume of oxygen; the share due to the nitrogen is 0.8 and that due to the oxygen is 0.2 .

At ordinary temperatures and pressures $18 \mathrm{~cm}^{3}$ of pure nitrogen and $35 \mathrm{~cm}^{3}$ of pure oxygen form saturated solutions with 1 litre of water. The quantities of each dissolved from air are therefore :

$$
\begin{aligned}
& \text { Nitrogen : } 0.8 \times 18=14.4 \mathrm{~cm}^{3} \text {. } \\
& \text { Oxygen : } 0.2 \times 35=7.0 \mathrm{~cm}^{3} \text {. }
\end{aligned}
$$

The air dissolved in water will therefore contain, roughly, 33 per cent of oxygen, and be twice as rich in this gas as atmospheric air. Laboratory tests have shown that the quantity of oxygen dissolved in water varies slightly with the nature of the water; distilled water absorbs more than fresh water, and soft water more than hard water. Distilled water absorbs $7.8 \mathrm{~cm}^{3}$ per litre at $20^{\circ} \mathrm{C}, 2 \cdot 1 \mathrm{~cm}^{3}$ at $100^{\circ} \mathrm{C}$. The water supply of Paris contains $6.06 \mathrm{~cm}^{3}$ per litre at $20^{\circ} \mathrm{C} ., 4 \cdot 3$ at $55^{\circ} \mathrm{C}$. and $2 \mathrm{~cm}^{3}$ at $100^{\circ} \mathrm{C}$.

When water is heated, supersaturation occurs. Nothing is given off up to a temperature of $75^{\circ} \mathrm{C}$. at which bubbles begin to form and rise until boiling takes place, when the bubbles are disengaged continuously. Air is only driven off continuously when the water is agitated incessantly from the commencement. When water that has been boiled is cooled, the inverse takes place; a litre of water 
absorbs, for instance, $2 \cdot 3 \mathrm{~cm}^{3}$ at $44^{\circ} \mathrm{C}$. and $3 \mathrm{~cm}^{3}$ at $20^{\circ} \mathrm{C}$. If the water is agitated in air the false state of equilibrium is disturbed, and the water becomes saturated up to $6 \mathrm{~cm}^{3}$ of oxygen, Fig. 9 . The straight line is the normal solubility obtained when the water is continuously agitated, and the curved line gives the contents at differunt temperatures without agitation.

Thus conditions of unstable equilibrium may occur which cannot exist when the liquid is agitated, and in every case equilibrium is

FIg. 9.

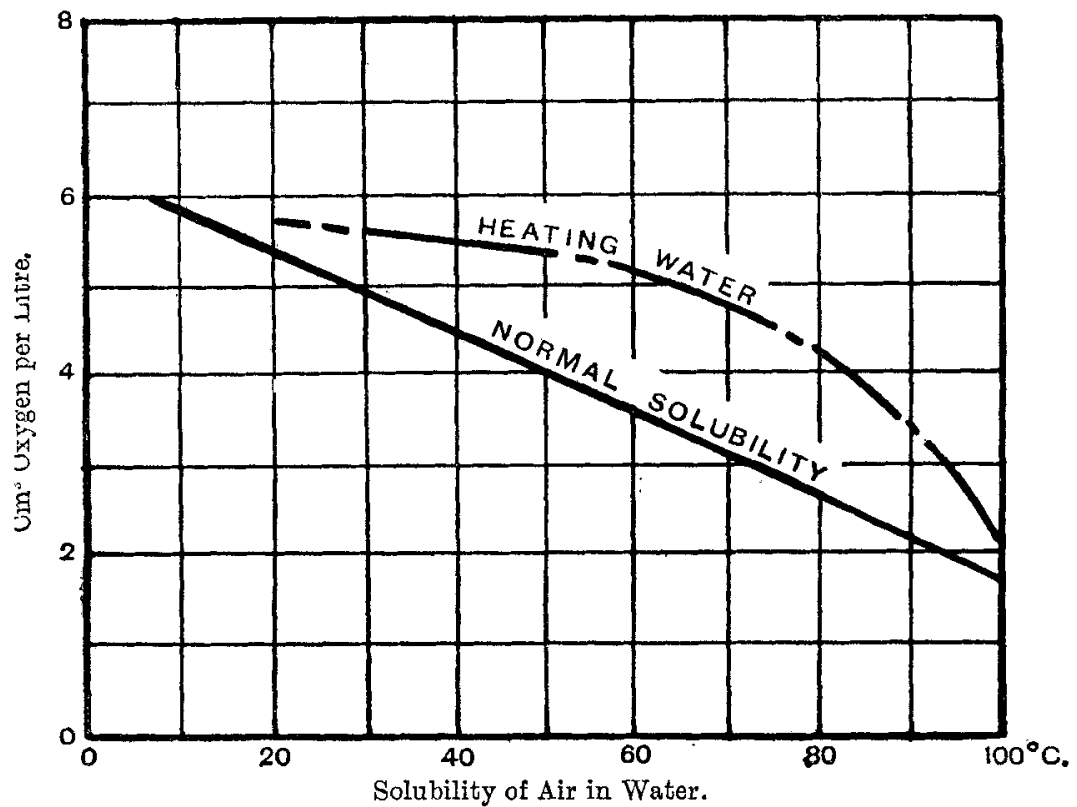

obtained for the particular temperature and pressure considered. If water is contained in a deep vessel, a sample taken at any moment from the surface contains the same percentage of gases as a sample taken from below; the diffusion of oxygen in water is extremely rapid. The area of the surface exposed to the air of water that has been boiled has an influence on the rate of absorption. Thus the water resulting from the condensation of the vapour in the air 
contains $4 \mathrm{~cm}^{3}$ of oxygen per litre at $60^{\circ} \mathrm{C}$. The same quantity of water of condensation exposing only a small surface to the air contains only $3 \mathrm{~cm}^{3}$ of oxygen per litre at the same temperature. According to Hohn, the surface affects the rate of absorption of the oxygen, as is shown in curves I and II obtained with a surface II (seven times that of the surface I), Fig. 10.

EIG. 10.

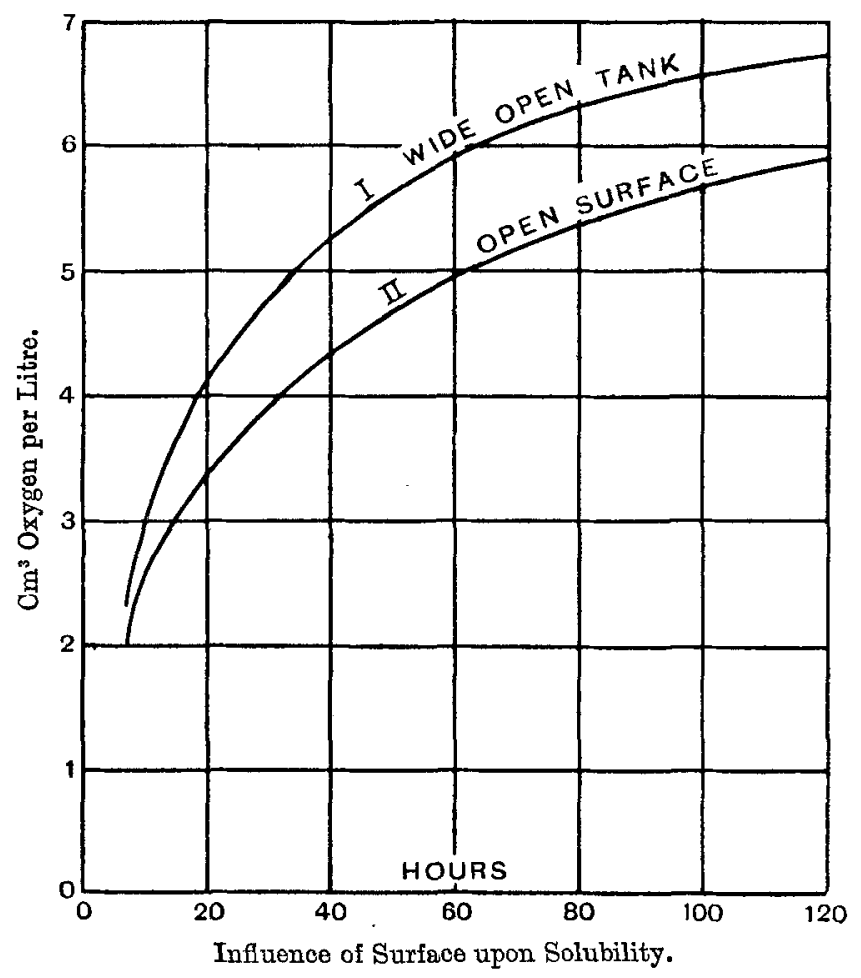

The possibility of changing the air also has an influence. If the reservoir is closed, but not hermetically, the oxygen is absorbed less rapidly than it could be from the open air, in which case saturation would be almost instantaneous, owing to the contact and the movement of the water. Hohn gives the following curves, Fig. 11 . 
Method of Obtaining the Oxygen Content.-All the methods of analysis that have enabled the data given above to be obtained and the determination of complete absence of gas have been made by Max Müller's method in which certain modifications have been made.

Fig. 11.

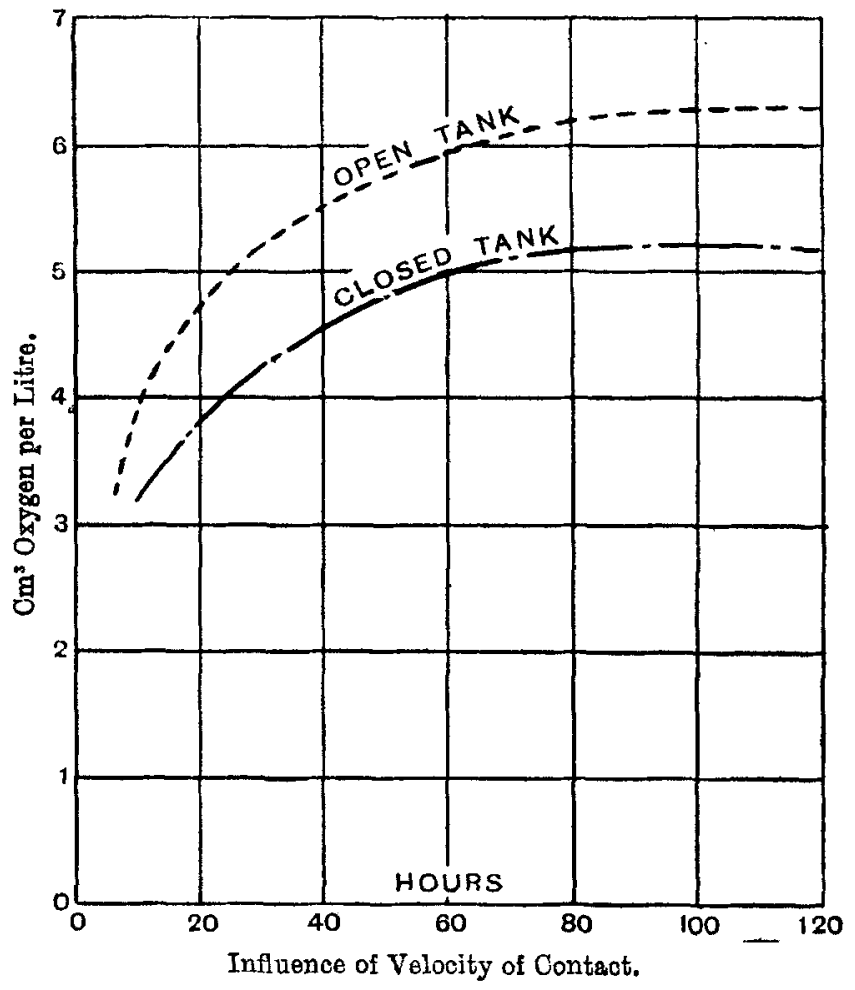

General Description.- The water is collected in a vessel protected from the air. Manganese sulphate is dissolved in it, and by the addition of sodium hydrate the hydrate of manganese monoxide is precipitated; this absorbs the oxygen contained in the water, transforming it into an oxy-salt and the dioxide. This is treated with hydrochloric acid, which reacts on the oxides of manganese, 
giving off a quantity of chlorine equivalent to the quantity of oxygen absorbed. The freed sodium chloride reacts on potassium iodide and sets free the equivalent of iodine. Titration by hyposulphite of soda gives the quantity of iodine and enables the equivalent amount of oxygen to be determined.

Method of Operation.-It is important, when it is a question of determining the quantity of oxygen in water containing but little, to avoid any contact of this water with the air. For this it is only necessary to put a small quantity of parafin at the bottom of the 2-litre flask in which the water will be received and to dip the tube bringing the water below its surface. Thus, during the whole operation, an insulating covering will be provided over the surface of the water.

After the water has been collected in the flat-bottomed 2-litre flask, 1 gramme of sulphate of manganese is added and the neck is immediately closed with a rubber stopper fitted with a funnel having a stop-cock and a glass tube connected by a rubber tube to another glass tube of larger diameter, carried on a stand.

On the principle of connected vessels, it is possible, by raising or lowering the tube, to change the pressure in the flask and to cause it to suck in liquid contained in the funnel fitted with the stop-cock. It is necessary that there should be no air bubbles in the apparatus, and this is easily attained after a little practice. When the sulphate of manganese has been dissolved, $5 \mathrm{~cm}^{3}$ of sodium hydrate in solution is introduced through the funnel of the stop-cock and the flask is shaken. A dirty white precipitate is formed, turning more or less yellow, according to the quantity of oxygen contained in the water. At the end of a quarter of an hour the absorption of oxygen is complete.

The next operation is to introduce $2 \mathrm{~cm}^{3}$ of solution of 50 per cent of iodide of potassium free from iodate through the funnel fitted with the stop-cock, and then to add $20 \mathrm{~cm}^{3}$ of concentrated hydrochloric acid. The flask is shaken until the precipitate has dissolved; the liquid takes a yellow colour, darker as the quantity 
of iodide freed is greater. The flask is then emptied into a bath and the iodine determined by titration.

The most practical method consists in preparing a standard solution of iodine containing 22.74 grammes per litre; that is, of such strength that a cubic centimetre corresponds to a cubic centimetre of oxygen. A solution of hyposulphite is prepared of such strength that it corresponds practically volume for volume with the solution of iodine. The number $\mathrm{N}$ of cubic centimetres of this solution corresponding to $20 \mathrm{~cm}^{3}$ of iodine is determined experimentally. To the solution of iodine contained in the bath $\mathrm{N} \mathrm{cm}{ }^{3}$ of hyposulphite is added, and then a small amount of starch, and the iodine is titrated again until a permanent blue colour is obtained. Let $\mathrm{N}$ be the number of cubic centimetres of iodine added. The iodine contained in the test will correspond to $20 \mathrm{~N} \mathrm{~cm}^{3}$ of liquid titrated, and the test will consequently contain $20-\mathrm{N} \mathrm{cm}^{3}$ of oxygen.

This method is simple, rapid and exact. It is delicate in application in the case in which the water contains traces only of oxygen, on account of the avidity of hydrate of manganese, which absorbs instantaneously the oxygen of any bubble of air coming into contact with the water. It is necessary to ascertain that all the reagents are pure, and particularly to make a blank test of the hydrochloric acid on iodide of potassium.

Removal of the Gases.-The elimination of the oxygen dissolved in water may be effected either by physical methods, or by chemical methods, or by the two combined. The principal physical methods to be mentioned are: agitation of the water; the action of temperature; and the action of vacuum. These three methods may be used either separately, or in the majority of cases in combination, in order to obtain the elimination of the gases from the water. Of the chemical methods the only one to be considered is the action of highly oxidizable iron, which absorbs the oxygen from the water before entering the boiler.

Removal of the Gases by Physical Methods.-Agitation of the 
water is used in various appliances in the form of fine spraying or circulation through channels of varying section, or again by the action of points and roughnesses on the current of water. These can only have the effect of preventing supersaturation at the particular temperature and pressure. Consequently they can have no effect beyond that of the action of temperature combined with a vacuum. Raising the temperature diminishes the solubility of the gases. But even if the temperature of the water is raised to $100^{\circ} \mathrm{C}$., the solubility cannot be neglected ; solutions containing about $2 \mathrm{~cm}^{3}$ per litre can be obtained at atmospheric pressure. The operation of heating, however, produces by itself a great improvement in the condition of the water entering the boiler, for there is no longer such a large release of air when the feed-water comes into contact with the water at a higher temperature in the boiler.

A vacuum would give better results still if it could be carried out at a very high temperature, the solubility of air being proportional to the pressure, according to Henry's law, as has been already stated. But the vacuum, particularly when the water is hot and attains $80^{\circ} \mathrm{C}$., cannot be very high, for otherwise the water would boil. At $80^{\circ} \mathrm{C}$. it is not possible to fall below about 0.5 atmosphere, and at $60^{\circ} \mathrm{C}$. more than 0.8 atmosphere.

If the three methods mentioned are used in combination, that is to say, if the water is submitted to the combined action of temperature and a vacuum, and supersaturation is prevented by mechanical action, the results shown in the following diagram will be obtained, Fig. 12 (page 316).

This curve has been prepared and published by a large German company making an apparatus, which uses the three methods simultaneously. It appears to be, if examined carefully, favourable to the use of these methods. Notwithstanding this, the firm is obliged to state: "There are still some milligrammes of oxygen that cannot be removed by a very high vacuum and by reheating the feed-water, but they have no aggressive action; the danger arises alone from a rich supply of oxygen."

The action of iron on dissolved air in the feed-water enables 
even the last traces of oxygen to be removed and water to be obtained absolutely free from oxygen. This proves that even the "last

$$
\text { Fir. } 12 .
$$

Absorption of Oxygen in Water at Different Temperatures and Vacua. Milligrammes of Oxygen per Litre.

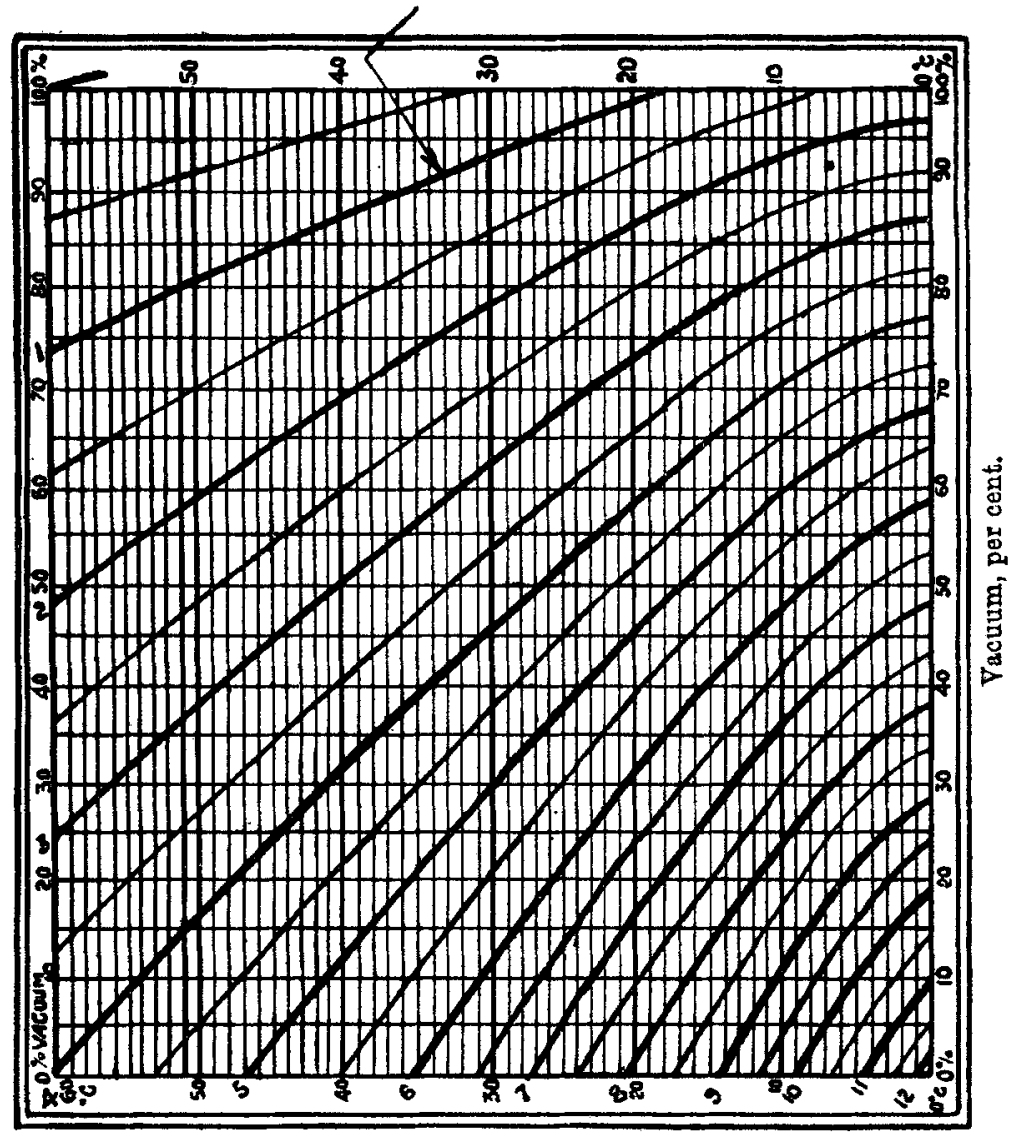

Temperature.

milligrammes " of oxygen are capable of causing rust and corrosion. Nevertheless, the physical operation of removing gases gives very satisfactory results, because it diminishes the percentage of oxygen 
in the feed-water and slows down the corrosion. But the use of a vacuum appears very difficult in appliances of large sizes, as it requires a very keen supervision at all points; and when leakage occurs, there is a churning effect produced in the water by the entry of the external air which, notwithstanding the decrease of pressure caused by the air temperature, produces the opposite result to that which is desired. A leakage of this kind is hard to locate, and for this reason it is always possible that such leakages may exist.

Numerous appliances have been constructed in England, America, and in Germany, for effecting physical removal of the gases. The principal ones in use are the following :-

(A) Thermal and Mechanical Removal of Gases.-All feed reheaters open to the air produce a removal of gases in so far as they diminish the solubility of air in water. Those in which the water flows in contact with steam in reservoirs that are not tight are those which best perform the operation of the removal of gases by heat alone. Those that reheat the water in closed receptacles, 0.g, in tubular economisers, remove the gases to a great extent from the water, but introduce gases into the boiler when it becomes necessary that the gases that have been removed are allowed to escape. Among these appliances those most in use are superheaters by Cochrane and Webster, and Weir, etc.

The apparatus of Chevalet, by its construction as a superheater with plate surfaces, differs slightly from the preceding appliances, and in this the steam bubbles through the water. All the "heating nozzles" of apparatus in which water is subjected to mechanical action and to the action of heat come within this class.

These appliances are accessories in the operation of removing the gases; there is one which can lay claim to the production of a better result, and that is the Aerex apparatus, in which the water loses its gases in being divided over two rubbing surfaces. The gases reunite and are removed by a valve when they have attained a sufficient quantity. 
The Siegmon Schmitt gas remover adopts a principle similar to that of partitions with baffles. The two appliances are placed on the delivery; they have more effect when the water is hot, but, on the other hand, they suffer some reduction in their action in consequence of the pressure on the feed-water, the pressure assisting the solution of the gases.

To eliminate gases by heat the firm of Balke, in Germany, combines an arrangement for reheating the water by steam with extraction of the gases evolved in an appliance in which the water is subject to forced circulation, and the air removed is exhausted by an arrangement of valves at the top of the apparatus. The water freed from gas is kept in contact with a layer of inert gas at the lower part of the apparatus. This somewhat complicated installation does not appear to have met with much success.

(B) Vacuum Appliances for Removing Gases.-Some firms have constructed apparatus using a vacuum for removing gases from water. Most of these are schemes of apparatus which have but rarely been developed into practical shape; such are the arrangements of Meihof worked by Halvor Breda and the Griesheim Elektron Lauta. The "Deutsche Sanitäts Werke" of Frankfort have constructed apparatus which is of considerable interest.

The Meihof, applied at Swinemunde, acts by passing the water from which the gases are to be removed through a siphon arrangement. If the branches of the siphon are of sufficient length, a vacuum is produced at the upper part, due to the suction in the descending branch. This vacuum causes the release of gases dissolved in the water at the top of the siphon. The principle is very simple and requires no mechanical arrangement; the water from which the gas has been removed is returned to the level of the water supply. The vacuum obtained in such an arrangement is not very high; it is limited by the destruction of the vacuum in the siphon by accumulation of gases. When these gases are removed, the vacuum disappears; this removal is produced by valves arranged on the particular part of the siphon operated at the time 
when the water returns for priming the siphon. In the apparatus constructed at Swinemunde for the removal of gases, the maximum vacuum corresponded to 5 metres (16 feet) of water, which is not a high degree of vacuum.

The apparatus of the Griesheim Elektron Lauta, is of very simple construction, consisting of a reservoir in which the temperature of the water is raised by means of steam. A vacuum is created by the heated water and the gases that accumulate are extracted by means of a pump.

The apparatus made by the Deutsche Sanitäts Werke is largely in use and is of very original design. In principle it consists of a tube with numerous contractions, to the upper part of which a vacuum pump is attached. At the base of this tube, which is immersed in the water from which the gases are to be removed, there is an adjustable air suction-pipe. Doubtless this air passes over an oxidizable filter in order that its oxygen may be removed from it. As a result of the vacuum, the water and air that have been filtered rise in this column; there is a vigorous shaking of the liquid due to the passage of the gas and to the contractions. This violent movement in conjunction with the vacuum causes the release of the gases dissolved in the water, and these gases are drawn into the upper part of the apparatus by the vacuum pump. It is certain that different conditions prevent a high vacuum from being obtained, in particular the shaking by use of gases and the height of the apparatus, which is far from attaining the dimensions required by the barometer. For this reason it is said "that there are still a few milligrammes of oxygen remaining in the water"; nevertheless, this apparatus certainly removes, the oxygen to a great extent from the water that has passed through it, and it contains at its maximum the percentage corresponding to saturation at the pressure obtained in the column and at the temperature of the water. This apparatus appears to be in wide use.

Chemical Removal of Gases.-All chemical methods of removal of gases in actual use are based on the same principle. They consist in causing the water that it is desired to free from oxygen to pass 
over iron. If the iron has a sufficiently large surface, if its chemical composition is carefully selected in order that it may be very easily oxidizable, and, lastly, if the water is circulated sufficiently slowly for the oxidizing action to be complete, the chemical removal of

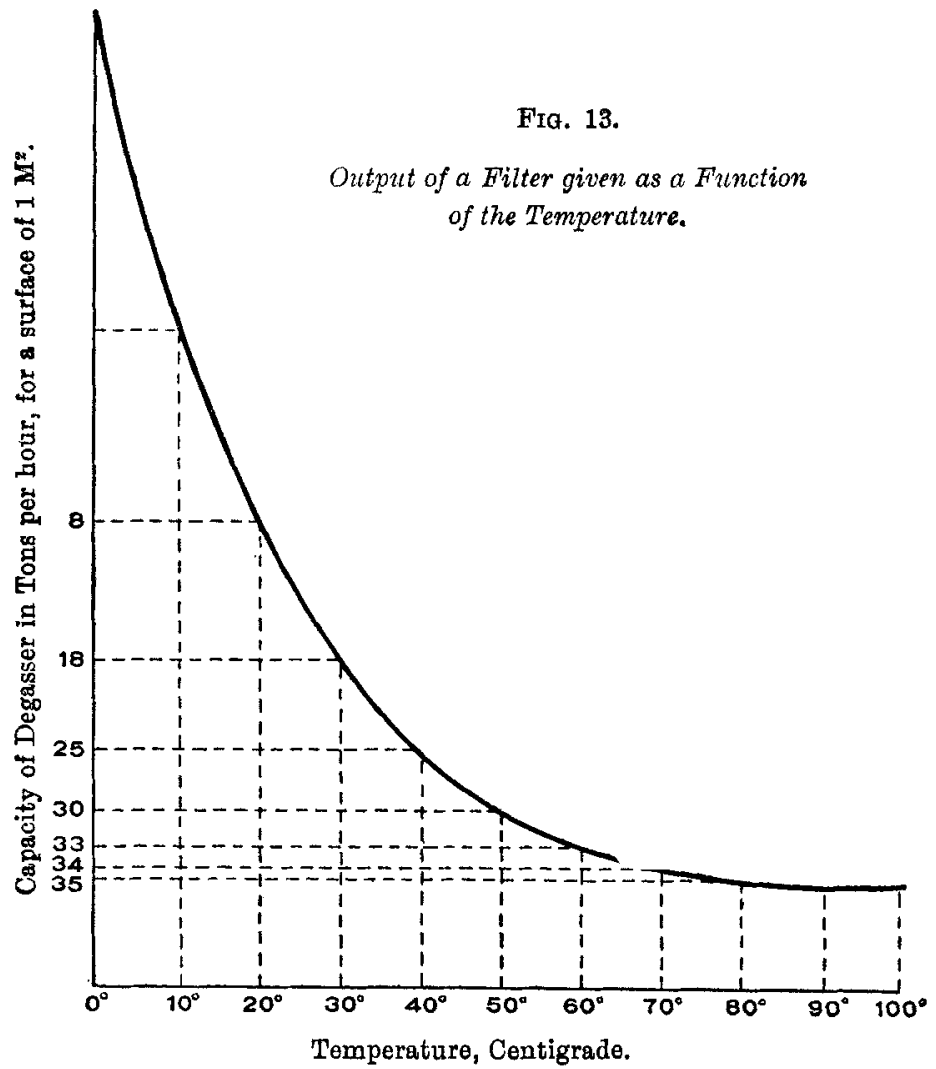

the gases enables all the oxygen contained in the water to be eliminated; in fact, the oxygen may be combined with iron in the presence of water so as to give different hydrates, of which the richest is rust :

$$
2 \mathrm{Fe}+3 \mathrm{O}+3 \mathrm{H}_{2} \mathrm{O}=2 \mathrm{Fe}(\mathrm{OH})_{3}
$$


This reaction is practically quantitative; it requires more or less time according to the temperature at which it takes place, but it can always, whatever be the temperature, effect a sufficiently complete removal of the gases, if the iron is largely in excess in regard to the oxygen, so that nothing but minute traces of gases remain in the water.

For the same quantity of iron turnings, the quantity of water from which the gases are removed is proportiongl to the speed of the reaction, and consequently proportional to the temperature. The output of a filter is given as a function of the temperature by the following curve, Fig. 13. If the velocity is caused to vary in a given filter, it will be seen that it cannot yield more than a. certain limited quantity if it is desired that the removal of the gases should be efficient.

The output is also proportional to the quantity of turnings in contact with the water; this quantity, moreover, cannot be increased indefinitely because the size of the apparatus would require to be sufficiently reduced so as not to be an obstacle to its introduction into a boiler room.

Another factor intervenes in the working of these appliances: it is the facility with which the metal used becomes oxidized. Practice shows that very impure iron rusts very rapidly. Laboratory experiments have enabled the Author to find a catalytic agent of great importance, namely, manganese. The presence of manganese hastens the oxidization of the metal; nevertheless, if this action shows itself in the case of small percentages corresponding to the manganese steels, it is not so well marked in the case of higher percentages. A metal which is rich in manganese is cast-iron, and cast-iron is much less oxidizable than steel. The physical and chemical composition of the metal has therefore a great influence.

The catalytic action of manganese may be represented by the following curve, the metals under consideration being very closely allied in composition and physical properties, and differing only by their percentage of manganese, Fig. 14 (page 322).

To sum up, it is seen that from the chemical point of view, the removal of gases by iron depends : on the surface of the metal, on 
the temperature of the water, on the speed at which it passes over the metal, and, finally, on the composition of the iron.

To obtain a maximum surface with a certain quantity of iron it must be used in a finely subdivided form, preferably in turnings. There is then a physical removal of the gases at atmospheric pressure which reduces the gases in the water to the point of saturation for air at the temperature and under the pressure in question. This action is of great advantage, and reduces the amount of turnings required to a considerable extent.

Fra. 14.-Velocity of Absorption of Oxygen for Degasser.

The Rates of Absorption of Oxygen are expressed in equal proportions for the same velocity of flow of water through the Apparatus.

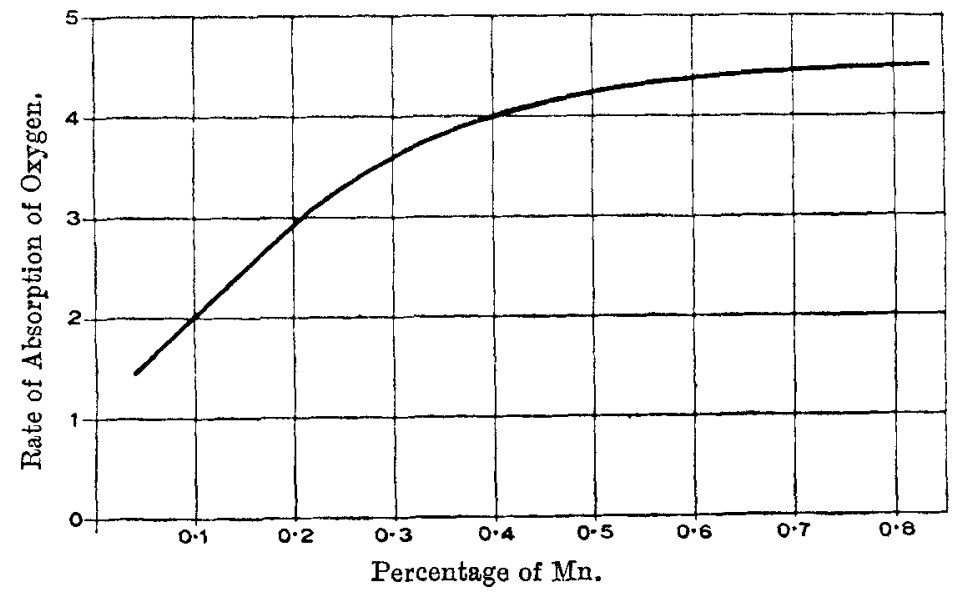

In commercial appliances, the following phenomenon may occur : When the coating of rust becomes very thick, contact of oxygen with the iron is no longer good, and the chemical removal of the gases may be considerably slower. Notwithstanding this, under the worst conditions, a water leaving such apparatus may be found to contain less than $0.5 \mathrm{~cm}^{3}$ of oxygen per litre. There are some other appliances working on the same principle as the German degassers constructed by the firms of Hulsmeyer and Seiffert, and an American apparatus due to Professor W. H. Walker. 
The Hùlsmeyer apparatus consists of a column of iron turnings distributed in baskets of perforated sheet-iron, which allow it to be distributed equally. This column of iron turnings ends in a filter which retains any particles of iron-oxide that may become detached. At the top of the apparatus there is fitted an automatic float-operated valve for the relief of the gases released by physical action. The water circulates from the bottom to the top of this very simple apparatus. The Hulsmeyer apparatus may be included as either falling under the suction type or the pressure type.

The Seiffert apparatus is constructed on the same principle, but comprises a reheater. Starting with the fact that heat encourages physical and chemical removal of the gases, Seiffert filled the tubes with a tubular superheater filled with iron shavings. The apparatus is of the most simple kind, and is, perhaps, very effective, but the replacement of the iron shavings in the tube cannot be a very easy matter.

Prof. W. H. Walker proposes an apparatus for removing the gases from hot water, consisting of a receptacle filled with perforated plates one above the other. This apparatus he styles a "deactivator." The only point that appears to have been overlooked is the absence of a valve for allowing the gases released in the apparatus by its mechanical action under the influence of the circulation of water between the plates.

To avoid the slowing of the oxidizing process by the rust deposit on the iron, the Kestner Co. has designed an apparatus based on the following: If an apparatus for removing gases by means of iron is left with the iron rusty, it is found that after a day of rest, the rust, which was red, has become an oxide of a greenish-black colour. This transformation is due to the reduction of the ferric hydrate under the influence of the iron; a saline hydrate of the oxide and $a$ ferrous oxide is obtained. The following is the reaction :

$2 \mathrm{Fe}(\mathrm{OH})_{3}+\mathrm{Fe}+2 \mathrm{H}_{2} \mathrm{O}=\mathrm{Fe}_{3}(\mathrm{OH})_{8}+2 \mathrm{H}$.

$2 \mathrm{Fe}(\mathrm{OH})_{3}+\mathrm{Fe}=3 \mathrm{Fe}(\mathrm{OH})_{2}$.

This reaction takes place in the middle of the reducing agent, 
that is, in the presence of iron and in the absence of oxygen. The saline oxy-hydrate formed is easily removed from the iron by washing, and moreover it is capable of further absorbing oxygen in becoming ferric-hydrate. In order that the apparatus for removing the gases should retain its working speed, it is necessary that it

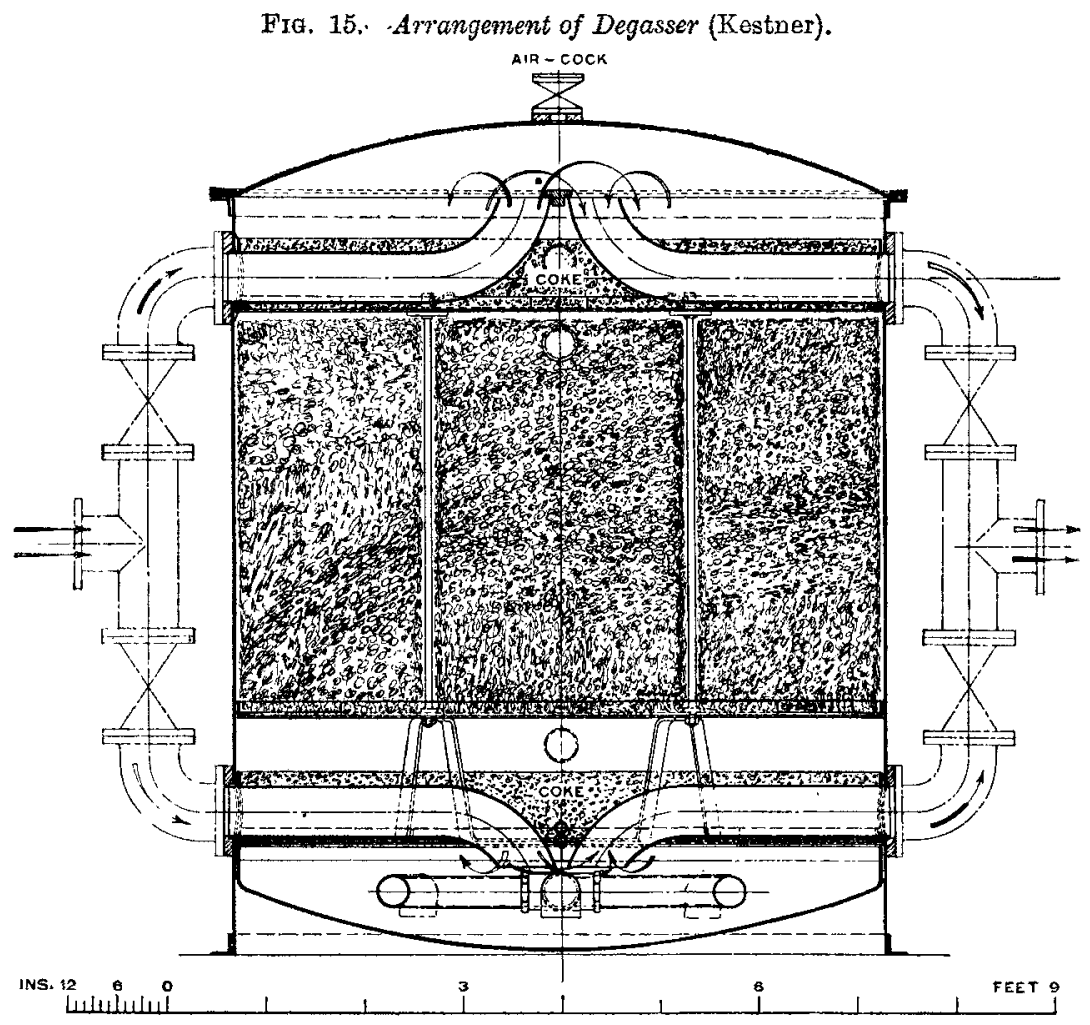

should be put alternately in and out of circuit, the two appliances in work retaining the power of the iron for absorption until the latter has been completely oxidized. The Kestner apparatus, Fig. 15, is based upon this principle, and consists of a column of turnings of sufficient quantity for half only to be in action, and to allow that the water shall have lost these gases on arriving at 
half the height. The second portion of the iron being in contact with this deoxidizing agent gives rise to rapid reduction, its rust changing to the condition of a suboxide. At the end of the day, the direction of flow is reversed. The water then passes over the iron, which has become a suboxide, which is then changed to a peroxide, and arrives free from gases on the surface (rusted the day before) which in turn is reduced. Thus, each half of the apparatus works alternately by inversion of the direction of circulation of the water, and the removal of the gases becomes continuous and absolutely regular.

The Kestner apparatus consists of a column of iron turnings placed between two filters with an intake and outlet for the water at each end of the apparatus, in such manner that the direction of flow of the water can be inverted at regular intervals. Like the other filters using iron turnings, it may claim completely to eliminate the oxygen from the water with greater certainty. The consumption of turnings and the speed of the absorption of oxygen are approximately regular, and the arrangement for allowing steam to be admitted at the base of the apparatus allows it to be cleaned by blowing steam through and removing any rust which may become detached from the iron on which it was produced.

The consumption of iron turnings in the apparatus for removing gases is very small ; according to the percentage of oxygen contained in the water, it varies from one to three grammes per cubic metre (per ton) of water which reduces the cost of the removal of gases to a negligible figure. The iron consumed in the gas-removing apparatus corresponds to the same weight of boiler-plate which has been saved from destruction, and the difference in first cost between the two materials destroyed forms a very great saving.

Whatever may be the method employed for removing the gases, it involves a circuit of water closed from atmospheric contact if it is to give a large output. The water which has been freed from gas can become saturated very rapidly by air. It is, therefore, advisable that the tanks in which the water may be retained should be as well enclosed as possible, and that on the whole of the pipe system there should be a small positive pressure. A head of a 
few centimetres of water is sufficient to prevent the entry of air which might happen accidentally through bad joints or accidental circumstances. In such a pipe system the only place at which the entry of air may be possible is the condenser in which the vacuum is produced. The tightness of the condenser should, therefore, be very carefully tested. Moreover, to avoid the effect due to any air entering by accident, which may always occur, it is essential that the water should pass through the gas remover immediately before entering the boiler, even though this water should result from condensation, and though it may only contain a small quantity of oxygen. Iron turnings are only consumed according to the percentage of oxygen in the water, and water free from gas does not change this ; consequently, it does not necessitate expense by being passed through the degasser. The degasser so placed protects the boiler and the economiser from any attack by the gases, and acts as a doorkeeper to prevent the entrance of anything undesirable.

The study of continuous blowing down, on the one hand, and that of degassing on the other hand, have led to the conception of a complete circulation system for a central station using distilled water. The main guiding principles of this idea are as follows :-

The ability to use any one boiler as a distilling apparatus, and to feed the other boilers with the water distilled in this boiler.

The blowing down of a large quantity of water from the boiler that is performing the function of distilling; the blowing off of only a small quantity of water from the boilers using distilled water, and the conveying into the distilling boiler of the water so blown down.

The protecting of the condensed water from all contact with the air by means of a closed circuit; the degassing of the condensed water before its passage through the economisers and the boilers.

From the moment at which the water is degassed the keeping of it enclosed so that no entry of air is possible.

The degassing of the water to be purified before it passes into the distilling apparatus.

There should be no point on the circuit of the purified water in common with any on the circuit of the distilled water. 
The only appliances that may be common to the boilers are the blow-off manifolds and the steam mains.

It will be seen that the scheme of this system of water circulation purification and degassing rests on a very broad basis, and that the distilling apparatus may equally well be an evaporator or a boiler. Each has its respective advantages.

For a water circulating, purifying and degassing plant the following system is proposed, Fig. 16 (page 328) :-

One of the boilers is to evaporate the purified water coming from the purifier. It will supply steam that will go with that generated by the other two boilers to the steam main. The steam will pass through the turbines, it will be, condensed and it will re-enter the boilers as distilled water after $\mathrm{jt}$ has passed through the degassing apparatus.

All the boilers are to be blown down, the boilers using distilled water to the extent of, say, $2 \frac{1}{2}$ per cent, and that performing the distillation of the water to the amount of, say, 10 per cent, and all of these amounts that have been blown down are to pass collectively through the purifier in which they will be subjected to the same treatment as the ordinary "make-up" feed-water, and the impurity is to be measured by the testing apparatus. The purified water is to be returned to the particular boiler performing the distilling, after it has passed through a special degassing appliance.

The boiler containing the water that is to be purified will thus furnish the supply to the boilers working with distilled water in the form of steam; there will be no common portion between the circuit of the purified water and that of the distilled water. It will merely be necessary to provide an overflow reservoir to enable any excess of purified water to be reintroduced into the circuit if such excess should be produced.

All the boilers can be fed either with purified water or with distilled water, which enables any desired boiler to be used for the work of distilling.

Such a circuit will ensure that a central station will have the best possible thermal balance sheet, and moreover a longer life for the 


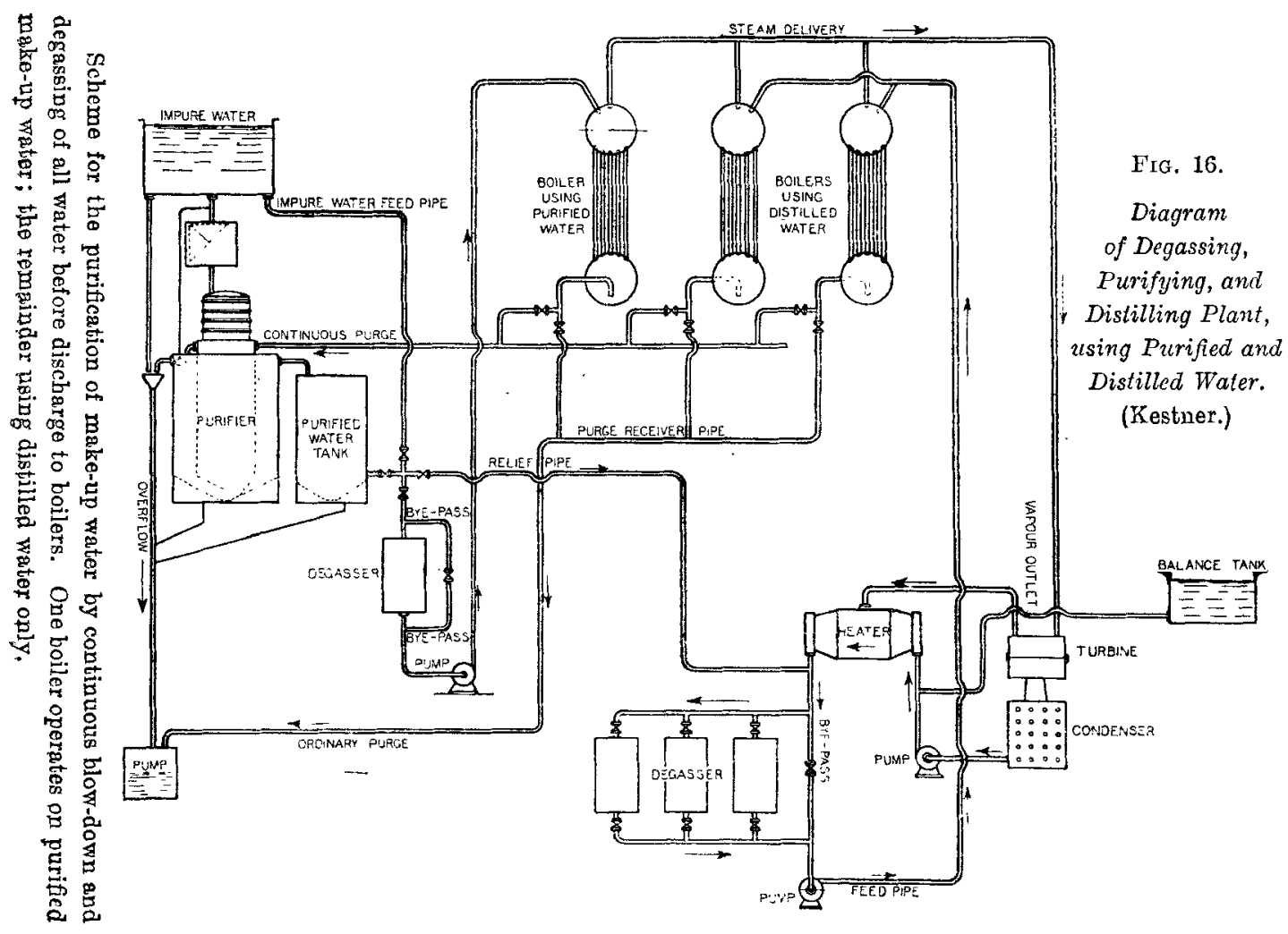

Diagram

of Degassing,

Purifying, and

Distilling Plant

using Purified and tilled Water.

tner.) 


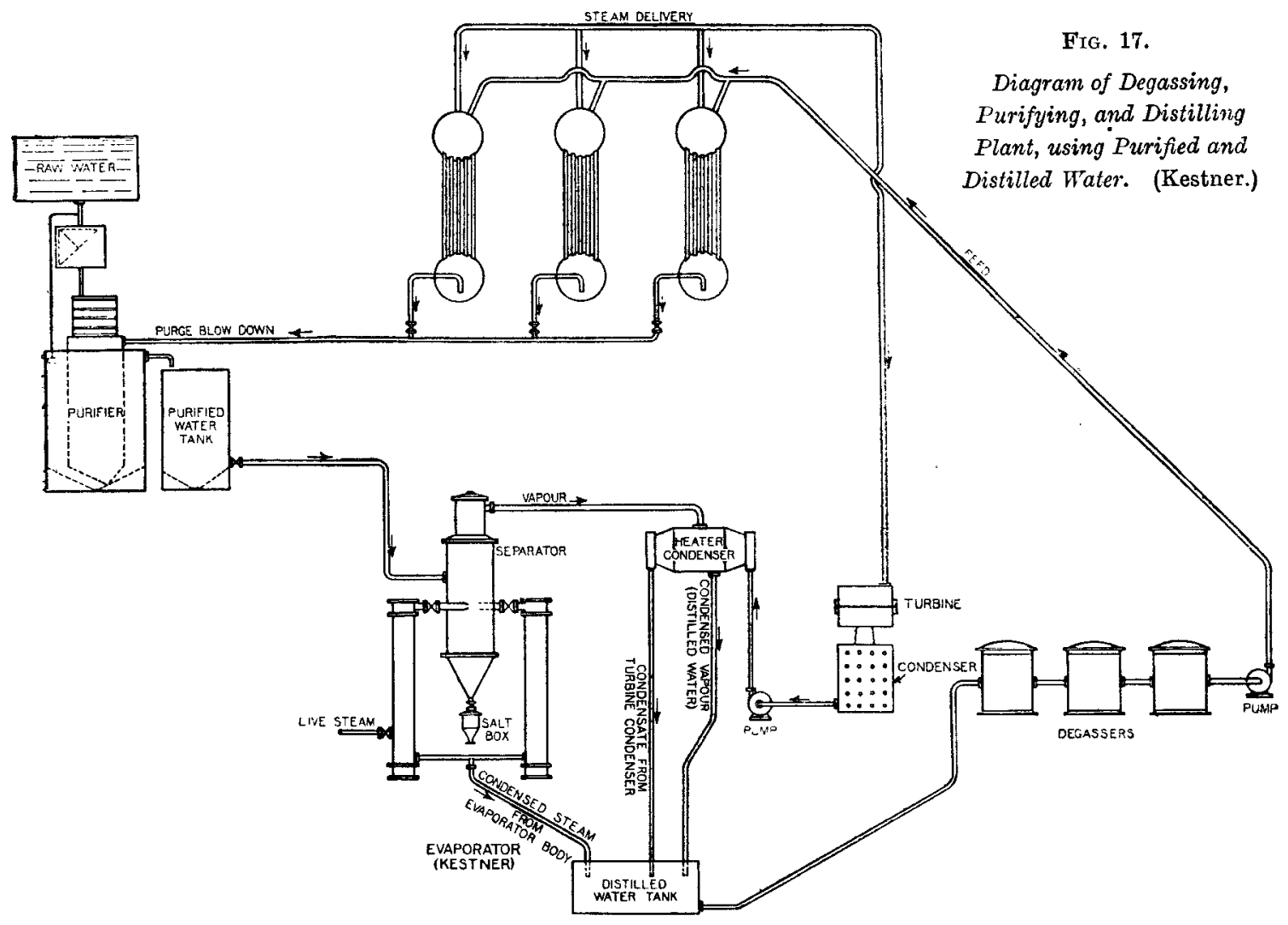


boilers, which the process protects from formation of scale and corrosion.

It is obvious that a similar scheme can be arranged for the distillation by means of an evaporator, which under certain circumstances offers advantages. Such an arrangement is illustrated in Fig. 17.

The Paper is illustrated by 17 Figs. in the letterpress.

\section{Discussion.}

The President said that, in view of the applause with which the Paper had been greeted, it was hardly necessary to propose a formal vote of thanks, and asked the Author to accept the thanks of the Meeting. The Paper brought very forcibly to his mind the necessity of an entente between Mechanical Engineers and Chemists. Chemists indicated what was wanted in regard to various operations, and it then remained for the mechanical engineer to devise the apparatus whereby those operations could be carried out.

The Resolution of Thanks was then put and carried with acclamation.

Sir William Pope, K.B.E., F.R.S. (President of the Society of Chemical Industry), said the pleasant duty fell to him, on behalf of the Society of Chemical Industry, to welcome the Author to the Joint Meeting of their two great Societies. The profession of chemical engineering had now become a well established one, and amongst its numerous members there were, as in every other profession, a few who stood out in that their work always bore the stamp of genius. They were accustomed to look for that mark of originality in everything the Author did. They had seen it in the past; they had discerned it in his Paper that evening, and they looked forward to seeing it again for many long years to come in his future work. Their recognition of the Author as an artist in a great profession was evidenced by the fact that the Society of 
Chemical Industry had conferred upon him the highest honour in their gift, namely, the Medal of the Society, and he was the only Frenchman who had received that award up to the present.

The Author had always been a great advocate of the necessity for closer co-operation between his own nation and this country, and it was because of his belief in that necessity that he had put himself to the inconvenience of coming to London to address the Joint Meeting of the Institutions. But he thought on the present occasion something more than that might be said. Captain Sankey had remarked on the necessity for more mutual effort between the chemist and the engineer, and in the present Joint Meeting there was to be seen, he hoped, the inauguration of a period of closer co-operation between the two great subjects with which they dealt. The alleviation of many of the troubles from which the world was now suffering depended upon progress in engineering, in chemistry, and in chemical engineering. It was no part of his task on the present occasion to open a technical discussion on the subject before the Meeting. There were many present who had a very wide practical acquaintance with the subject of the Paper, and he would therefore leave the discussion of the important points which had been raised to those who were better able to deal with them.

Professor J. W. Hinchlex, in opening the discussion, said the Author had been labouring at the subject a long time, and he believed he had succeeded in evolving a practical solution to a very difficult problem, namely, that of freeing water and steam raised from it from the corrosive oxygen and carbon dioxide usually present. The fact that ferric hydrate in contact with iron was slowly reduced to the ferrous state, and was again capable of performing the function of an oxidizer, was a remarkable chemical fact to be used in solving the practical problem. The absence of corrosion in the boiler would be not less important than its absence in other parts of the plant through the freedom from oxygen of the steam. The presence of air in steam was one of the bugbears of the chemical engineer. He did not believe the mechanical engineer worried about it, but he certainly had great evidence of it in the damage that was 
done to condenser tubes and other parts of his plant. He was very interested in the fact that the Author had found that castiron containing manganese was an extremely active rusting agent. He believed cold blast cast-iron liners were usually stated to be used in ordinary cylinders of steam-engines, but there was little doubt that many of those liners were cast from metal made in an ordinary cupola by the addition of steel containing appreciable amounts of manganese, and it was quite possible that much of the corrosion that was obtained in those cylinders might be due to the oxygen in the steam and the presence of manganese in the cast-iron. $\mathrm{He}$ believed manganese was a notorious agent for producing segregation, especially of sulphur as manganese sulphide. He would like to know from the Author whether he determined the percentage of sulphur at the same time that he determined the manganese, and whether the segregation of manganese had anything to do with those characteristic properties which he found, or whether it was merely manganese in solution in the cast-iron or equally distributed through it. He would also like the Author to say whether he found that the amount of graphitic carbon in the grey cast-iron which he used had any great influence.

Mr. Arthur Spyer said it was difficult for him to criticize the Paper, because he was so absolutely in agreement with the theories the Author had enunciated, especially in regard to the necessity of degassing feed-water. The Author had pointed out that carbonic acid gas and oxygen, when they were present in the feed-water, resulted in a recuperative action, because ferrous carbonate was first formed, then in the presence of oxygen this was transformed into ferric carbonate, which, being a very unstable body, again resolved itself into oxide of iron and carbonic acid gas, so that, if the carbonic acid gas was not got rid of, the action was continuous and recurrent. The Author had spoken at great length on the socalled chemical method of degassing, and he was very much interested to hear the propositions he made in reference to the use of what might be called the steel-shaving method, because until quite recently the results of degassing with that method of treatment had 
not becn altogether consistent; in fact, in many cases it tras known they had been unsatisfactory. There seemed to be several indications, however, that with the modifications suggested by the Author those difficulties might be overcome.

Another point raised was the use of evaporators. The vapour produced in ordinary evaporators frequently contained carbonic acid gas, oxygen, and traces of copper salts. If these substances obtained access to the boiler-water, they reproduced the gas difficulty and hence the necessity of getting rid of the gases. The most agreeable waters for drinking purposes were nsually impregnated with carbonic acid and oxygen, and whilst exceedingly pleasant to drink, they were exceedingly bad for boilers.

With regard to the suggested method of a continuous blowing out, he did not wish to be hypereritical in any way, but in a wellmanaged station at the present day, with the methods which were adopted of getting rid of solid matter in the feed-water, the amount of blowing out from the boilers should be very small. It was rather doubtful whether it would pay commercially to install somewhat costly apparatus as described in the Paper, unless very definite evidence were forthcoming of the gain to be obtained, because, after all, engineers unfortunately had to consider whether a particular apparatus paid or not.

In dealing with the subject of corrosion, the Author had reforred to galvanic action. In his own experience he had never yet seen a case in which he was convinced that galvanic action of itself had been the cause of corrosion. The wear of the zinc blocks which were frequently fitted in boilers, was more probably due to ordinary oxidation than to galvanic action. He was absolutely in agreement with the Author with regard to the beneficial effects of heating the feed-water before subjecting it to the discharge pressure; it undoubtedly helped to get rid of gas, and facilitated the action on the water of any chemicals which might be introduced. It reduced the solubility of the salts at high temperature, and therefore enabled a great proportion of the salts to be got rid of before they went into the boiler. It should be the object of engineers and chemists first of all to take care that no air got into the feed-water; in the second 
place, to take all possible steps to prevent the access of air to the feed-water subsequently, and, thirdly, to heat it as much as possible, so that any gases which were present might be eliminated so far as practicable.

Mr. R. W. BAILEY said he was sure that builders of steamturbines would be indebted to the Author for his valuable Paper, first, because it emphasized the need of removing oxygen from boiler feed-water, and secondly, it might be inferred that, if it was not removed, serious corrosion of the plant might result. Corrosion in a steam-turbine was a much more serious matter than in a boiler; and there was no doubt whatever that oxygen was responsible in many cases for blading decay. Although materials specially resistant to corrosion were employed in the construction of steamturbines, for example, 5 per cent nickel steel, high chromium steel, and special alloys, corrosion of blading might occur unless proper attention were paid to the feed-water. The firm with which he was engaged, namely, the Metropolitan-Vickers Electrical Co., had experienced cases of blading corrosion, and its research department had investigated the problem of water treatment with a view to its prevention.

The action of an iron deoxidizer of the type described by the Author was investigated, and the results obtained confirmed his remarks regarding its effectiveness. At first it was thought that iron turnings would meet ordinary requirements, but the idea was abandoned as it was feared that the water would traverse the mass in lanes, and uncertain and irregular action would result. $\mathrm{He}$ would like to ask the Author whether trouble of this kind was experienced with iron turnings. Attention was therefore directed to the use of expanded sheet steel, and Fig. 18, based upon experiments made with a small deoxidizer, showed the relation between the amount of iron, rate of flow, and oxygen removed as a percentage of that initially present. The action of such a deoxidizer was also investigated from a theoretical standpoint, in order that rational formulæ might be employed which could be used with some degree of confidence outside the range of the experiments. This was done 
MAFCH 1921. PURIFICATION OF BOILER FEED-WATER.

in Fig. 18, which gave values for cases up to 500 sheets, although the experiments were only conducted over one-tenth of this range. It was found that the rate at which the iron absorbed oxygen was rather more than proportional to the concentration of oxygen (this might not be absolute, but might be really due to a more extended

FIG. 18.

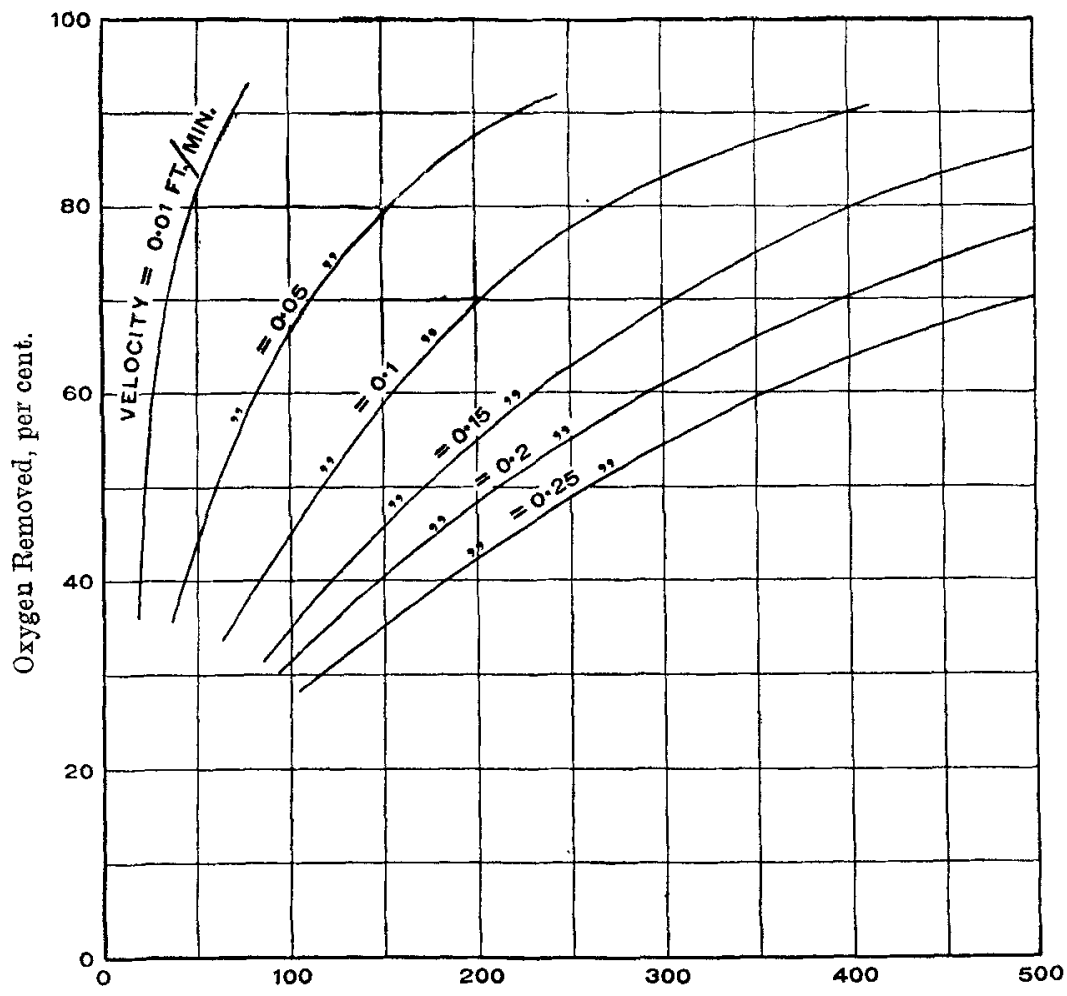

Number of Pieces of Expanded Metel (Surface area $=1.04 \times$ plane area).

surface being offered where oxidization was most active), and increasing the velocity of flow increased the rate of absorption at the iron; but, as the quantity entering was proportional to the velocity, the percentage of oxygen removed from the water decreased with increasing rate of flow as would be expected. The curves showed 
that the increase in eficiency with decreased velocity was appreciable. A rational formula taking into closer account the effect of concentration and velocity was not convenient for practical purposes, and the following approximate formula, which would satisfy most practical cases and was a basis of Fig. 18, might be given for the case where the water temperature was $15^{\circ} \mathrm{C}$.

$$
\log _{10} \frac{100}{100-P}=\frac{8 \cdot 1 S N}{100+1 \overline{2500 V}}+0 \cdot 04
$$

Where:-P = Percentage of oxygen removed.

$\mathrm{V}=$ Velocity of flow in feet per minute, based upon the gross cross-sertional area.

$\mathrm{N}=$ Number of sheets of expanded sheet steel.

$\mathbf{S}=$ Ratio :- Area of actual iron surface

The influence of temperature in increasing the rate of oxidation was most marked. Their information on this point was not very extensive, but it appeared to support the rough rule that it doubled for every $10^{\circ} \mathrm{C}$. increase in temperature. He would like to ask the Author whether such a relation agreed with the curve exhibited by him.

He was most interested in the alternating flow arrangement developed by the Author in order to take advantage of a regenerative action. His firm had developed a similar arrangement for a different reason, namely, that the rate of corrosion was so much greater at the inlet end that, to secure something like uniform oxidation of the iron throughout the deoxidizer, it was necessary to employ the alternating flow principle. It should be mentioned that the rate of oxidation at different positions in a deoxidizer of the kind considered was given approximately in Fig. 18 by the ordinates of the different curves measured from the 100 per cent line.

The Metropolitan-Vickers Electrical Co. did not make deoxidizers, but they had looked into the question of their action and design in order that, where necessary, recommendations could be made to customers in connexion with the steam-turbines supplied. Fig. 19 
showed diagrammatically a scheme recommended in the case of a large electric power-station in this country where corrosion had. been traced principally to dissolved oxygen in the feed-water. Only the make-up water was passed through the deoxidizer, and this flowed under gravity from the storage tank, $A$, via the pipe $B$ and

F'IG, 19.-Diagram of Feed. Water De-oxidation Plant.

(Metropolitan-Vickera Elec. Co.).
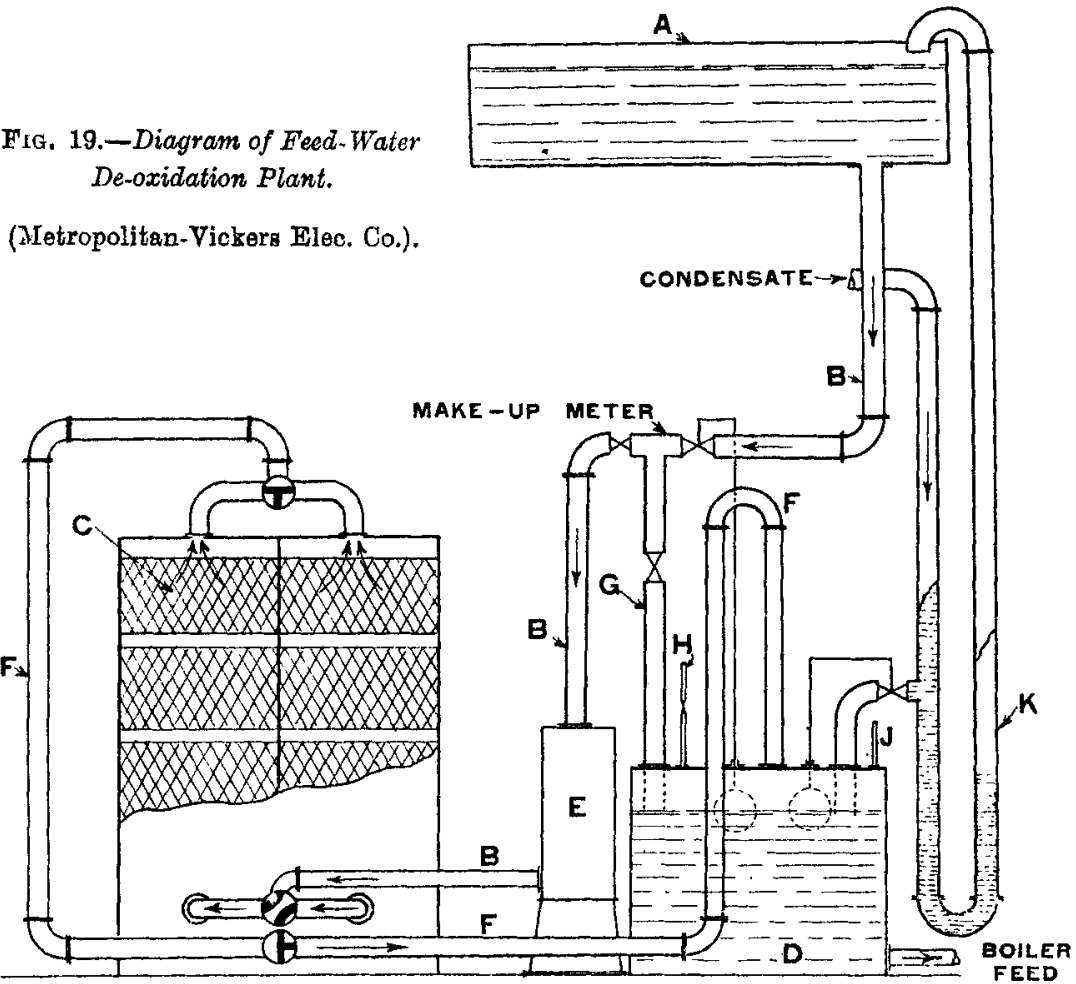

thermostat controlled heater $\mathrm{E}$, to the deoxidizer $\mathrm{C}$, its rate of flow being controlled by a float in the hot-well $\mathrm{D}$. The valve arrangements for reversing the direction of flow and for permitting one side to be opened up while the other was in operation, thus enabling replacement of the expanded metal to be carried out without stopping the plant, as also the arrangement for shutting 
down the deoxidizer completely, would be understood from the Figure. To prevent subsequent aeration of the make-up and of the condensate, the hot-well $D$ was steam-sealed, exhaust steam from auxiliaries being led in at $\mathrm{H}$ and its supply adjusted so that a small escape took place at the vent-pipe $J$. To prevent aeration of the condensate and at the same time permit a simple delivery to the overhead storage-tank when the level in the hot-well closed off its flow thereto, an inverted siphon $\mathrm{K}$ was employed and the branch to the hot-well was "teed" off the inlet limb as shown A column of "dead" water existed below this connexion, and thus sealed the condensate passing to the hot-well from the atmosphere. The delivery pipe from the deoxidizer was carried up as shown at $F$, in order to keep the upper part of the deoxidizer under sufficient pressure to enable a snifting valve to release any gas that might accumulate when heating was employed. (See also page 342.)

Professor F. G. Donnan, F.R.S., said he remembered that some years ago the question of the degassing of the water in the Calgoorlie water supply main, which had a very great length, arose, and he understood it was done by spraying at a reduced pressure. That was a very important question in connexion with conducting water over long-distances and for general questions of municipal supply. 'It would be of interest if the Author could state how that method could be developed for general supply purposes. It was quite impossible to use a chemical method on a very large scale like that, and it would therefore be of interest to know if it was possible to use the physical method successfully. He thought that spraying by nozzles at a sufficiently low pressure-vacuum would not remove the oxygen sufficiently, but that was not a matter on which he possessed enough knowledge to give a valuable opinion.

With regard to the Author's scheme of a boiler acting as a water distiller, he remembered inspecting in South America a boiler system which worked in very nearly the sume way. One boiler took the make-up feed, which was softened in the usual way, and acted as a water distiller to the other boilers. The condensed water came back, and there was a partial attempt at systematic blowing down, but 
it was not very great and was not particularly necessary. The boilers were mostly water-tube boilers working at $200 \mathrm{lb}$. pressure, and the water distiller was a Lancashire boiler working at about 50 lb. pressure.

Professor J. S. S. Brame said he had been carrying out a good deal of work in connexion with boiler corrosion, and he was quite convinced that the question of oxygen in the feed-water was one of the utmost importance. The Author considered that the action of water and oxygen first produced ferric hydroxide, a similar action to that which took place in corrosion at normal temperatures. $\mathrm{He}$ (the speaker) was not sure, however, that the actions were similar in a boiler where the temperature was somewhere of the order of $200^{\circ} \mathrm{C}$. In the deoxidizing of water by iron, the interesting point was the reduction of the $\mathrm{Fe}_{2} \mathrm{O}_{3}$ in contact with the iron in the absence of oxygen. At the point of entry the $\mathrm{Fe}_{2} \mathrm{O}_{3}$ existed as a maximum, but by passing the water in the other direction a dark greenish-black compound was obtained. It was an action which had been very largely overlooked, although it was mentioned in the old text-books ; for instance, a very interesting account of that action would be found in Gmelin's Chemistry (vol. v, p. 185: 1851). The Author had postulated the formation of a hydrated magnetic oxide $\mathrm{Fe}_{3}(\mathrm{OH})_{8}$. He (Professor Brame) would like to know whether there was any basis of analysis for such a formula. It was most difficult to get consistent results for the analysis of any of those products of oxidation except the fully hydrated oxide. From most forms of rust a considerable proportion of a magnetic oxide conld be separated by grinding them up to a very fine state of division and flowing them by means of water past a powerful magnet. One was so accustomed to look upon it that the formation of ordinary $\mathrm{Fe}_{3} \mathrm{O}_{4}$, either as $\mathrm{Fe}_{3} \mathrm{O}_{4}$ or in the hydrated form, was only produced under special conditions that it was somewhat surprising to find that, if a plate of iron was left just awash in water, so that plenty of oxygen could get through, the red rust formed first of all, and that as the mass was built up there was formed underneath a darkish green or almost black compound which was strongly magnetic. Those were all 
reactions which related to the question of separating the oxygen from the water by the iron process, and in addition they must play a very important part in the whole question of corrosion. The solution of the problem lay very largely in finding out first of all what types of action were involved.

The Author had referred to magnesium chloride. It had had a lot of sins to bear and a lot of burdens to carry, but he was not sure that it was such a sinner as it had been made out to be. If the whole of the magnesium chloride remained in solution, he thought it was very doubtful whether that action of hydrolysis producing the hydrate and free acid actually did take place, but it had been shown by Hof that the development of free acid only became an appreciable reaction when the magnesium chloride was left as a deposit on the side of the vessel during evaporation.

Dr. W. R. Onmandy said that a considerable number of years ago he had had a great deal to do with the installation of watersoftening plants, and the experience which he had thus gained made him very cautious in expressing any opinion upon the reactions which took place. Particularly was this true regarding the mutual reactions taking place between oxygen and carbon dioxide in the presence of iron. Unlike Professor Brame, he thought that hydrolysis played an important part. A previous speaker stated that he did not find much evidence of electrolytic effects. It seemed to him that the extraordinary effects of local corrosion, spotting, in a boiler must to some extent be attributable to the heterogeneous nature of the metal which was employed. He thought most of the members who had had a good deal to do with boilers would remember occasions when an old boiler, made in the days when puddled iron was used, resisted all the waters used in it and gave no signs of corrosion, but when that boiler was displaced by a steel boiler then entirely different results were obtained, even with the same water which had been used successfully in the wrought-iron boiler. It seemed to him that that might be attributed to the fact that the steel almost invariably contained segregations rich in manganese. It was known, not only from the experiments which 
had been described in the Paper, but from other work that had been done on the subject, that manganese was an extraordinary material for acting as a carrier of oxygen. It was mixed with potassium chlorate in order to get oxygen off, and manganese sulphate was added in many reactions because it acted as an oxygen carrier. In many ways it was an ideal substance for the purpose.

It was difficult to grasp the details of the Author's continuous process, but it was clearly intended primarily for power-stations and marine work where surface condensers were employed. He would like to point out, however, that more boilers were probably employed in producing steam for industrial operations where the condensed water could not be returned to the boiler. This led one to inquire as to the possibilities of the application of the de-oxygenizing process in its application to such boilers. It was a very fortunate thing that the curve for the rate of the degassing reaction was such that at a temperature of $90^{\circ}$ to $100^{\circ} \mathrm{F}$, a very considerable velocity had already been acquired, because that was about the temperature at which every engineer desired the water to go into his economisers. It might, therefore, be assumed that water could be heated by waste steam to $90^{\circ} \mathrm{F}$., sent through a de-oxidizing machine, and then passed through the economisers. Incidentally, eighteen months or two years ago, when getting quotations for economisers, he came to the conclusion that the makers were going to get over the difficulty by silver-plating them inside.

He would like to know whether the Author could say anything about the influence of traces of alkali on the rate of the removal of oxygen from boiler feed-water, because, as the members were aware, it was almost impossible to soften a water by any of the ordinary types of lime soda softening plants to within anything below 7 grains per 100,000 without having a certain amount of free alkalinity. He had met with a case of natural water which was so pure that it contained only 2 parts of solids per 100,000 , but which seriously corroded steel boilers, and yet the addition of 2 grains per 100,000 of caustic soda inhibited that corrosion absolutely. He would like to know if the presence of free alkali in any way had that effect of stopping the absorption of oxygen in the degasser, or whether it necessitated the use of much larger degassers. 
Mr. William H. Patcheli (Vice-President) said that some of the members had been striving for a long time to obtain closer association between the engineer and the chemist, and that had been brought about on the present occasion. The entente cordiale had been much in evidence during the last five years, and it had led to surprising changes, and he was glad that through the good services of M. Kestner it had led to the Society of Chemical Industry joining with the Institution in the present Meeting. Both Sir William Pope and Captain Sankey had said it was the inaugural meeting of the two bodies. He understood that it also set a precedent for the Society of Chemical Industry, in that he did not believe they had ever dared to meet anybody else before! He hoped the result of the combination would be thoroughly satisfactory.

M. Paul Kestnjar, in reply, said he was much obliged to the members for the kind reception that had been accorded to him. He was glad to hear the President, Sir William Pope, and Mr. Patchell agree with the suggestion he had made that the engineer and the chemist should work hand in hand, and that it would be of very great benefit to their industries. He would reply to the discussion in writing.

The Meeting then terminated.

\section{Communications.}

Mr. R. W. Ballexy wrote, in reply to Professor Brame's question relating to the character of the oxide coat, that he was struck by the remarkable uniformity and consistency in the action of their experimental deoxidizer, as shown by a period of operation of two to three months with only short stoppages at intervals for inspection or alteration in the number of sheets. During these runs no spottiness in the oxide coating occurred, which was very uniform 
in colour, red on the outside and greenish black below and next the metal. Probably the uniform conditions of flow, that could be obtained with expanded metal, accounted for the absence of a patchy coat. It would be interesting to know whether spottiness had been experienced with iron turnings, and he would like to ask for the Anthor's experience on this point.

With respect to the physical method of degassing described by the Author, namely, by exposing water to a reduced pressure, the action, of course, depended upon the magnitude of the partial pressure of the gas in the atmosphere to which the water under treatment was subjected, and this could be arranged to be very low indeed, although the total pressure might be appreciable. Thus water exposed to an atmosphere of steam if finely divided would be degassed, because the partial pressure of gas there might be nil or very small. In appreciating the advantages of the Author's continuous blow-down hot purification system of treatment, it was to be noticed that the inlet feed-water underwent degassing in this manner. To exploit this method to the full extent, however, it was necessary to draw off the gas liberated in order to have a low gas partial pressure. He would like to ask to what extent this was done. Instead of spraying water into an atmosphere of steam, a similar result, though not so complete, would occur if steam was bubbled through water. This happened in steam-boilers. A bubble of steam formed, having initially a gas partial pressure nil or very small, and as it rose it had a degassing action upon the water at its boundary. This was going on throughout the whole mass of the water, and thus it was that a large proportion of the dissolved gases was conveyed to the steam space and away to carry on corrosion of the plant beyond. This action of steam bubbles was not generally appreciated.

Messrs. J. H. Coste and W. E. F. PowneY wrote that the general question of gases dissolved in water was discussed at length by one of the writers in 1917 and 1918.* Since then they had had occasion

* Jour. Soc. Chem. Ind. xxxvi, p. 846, xxxvii, p. 170. 
to investigate the nature and amount of both gases and solids dissolved in the water and associated with the steam in a large power plant. They were generally in agreement with M. Kestner, but on some points they were unable to confirm his views. The greatly increased velocity of the softening process when it was carried out at an elevated temperature was undoubted. They could not, however, think that many boiler waters contained so much calcium sulphate that it would be partially precipitated during the softening process. The solubility reached a maximum at about $40^{\circ} \mathrm{C}$., and at $100^{\circ}$ was about 120 per 100,000 of water.

The view that magnesium chloride was hydrolytically dissociated in dilute solutions with actual liberation of hydrochloric acid was, in their opinion, based on a misreading of Casaseca's Paper.* $\mathrm{MgCl}_{2} 6 \mathrm{aq}$ seemed to be the stable form to $116.67^{\circ}$ and between this and $181 \cdot 5 \mathrm{MgCl}_{2} 4 a q$, after his $\mathrm{MgCl}_{2} 2 a q$. See also Cribb (Analyst XXv, 1900, 169). As properly softened boiler waters were usually more alkaline than $\mathrm{P}_{\mathrm{H}}=8$, the question of free acid could hardly come in. They were unable to agree with the view that the Author appeared to hold that sodium carbonate alone was better for softening than a mixture of this and lime. Lime had the great advantage of adding no soluble salts to the water, of removing magnesia from solution, owing to the low solubility of magnesium hydroxide and of directly reducing the admittedly very small hydrion concentration due to carbonic acid. The corrosion of boiler plates or of turbine blades was undoubtedly due, as the Author stated, to dissolved oxygen. Its action, however, appeared frequently to be that of a depolarizer, removing the ferrous ion from solution and forming finally that oxide which was in equilibrium with water at high temperatures, $\mathrm{Fe}_{3} \mathrm{O}_{4}$. It was the presence of dissolved oxygen alone which allowed the action of carbonic acid or of saline electrolytes to be continuous.

They had found that water condensed in the very diminished pressure obtained in the condenser of a large turbine fitted with separate and efficient water- and air-pumps was almost free from

* Comp. Rend. 1853, xxxvii, p. 350. 
oxygen, whilst that from a condenser fitted with a single pump for gas and water might even be supersaturated. The almost air-free water took up a considerable proportion of oxygen-about 25 per cent of that required for saturation in flowing over a $\mathrm{V}$ notch and cascading down into the pipe leading to the feed-water heater. No doubt the turbulent flow was accountable for most of this, not the steady flow with unbroken surface over the $V$ notch. In feed-water heatcr and open hot-well the concentration of dissolved oxygen would be about 70 per cent of saturation. Some reduction, principally shown indirectly by rusting in the water, but also by the reduced proportion of oxygen relative to "nitrogen," * was found at the inlet of the economiser. The water at the outlet was difficult to sample satisfactorily, but the steam delivered to a turbine had been examined and found to contain much less oxygen than would correspond to the nitrogen in solution, showing a loss of oxygen in the economisers and boilers. Some carbon dioxide was also found.

The writers' experience with condensers fitted with efficient air-pumps convinced them that, at least as a counsel of perfection, the soundest way of securing water free from dissolved oxygen was to allow no opportunity for the water, which could be condensed practically gas-free, to absorb any. That is, to use as far as possible a closed circuit. If the make-up water was kept down to a minimum, suitable chemical or physical means, which might be such as the Author suggested, could be used for degassing it.

M. Kestner appeared to state that water at $100^{\circ} \mathrm{C}$. and atmospheric pressure still retained a considerable proportion of oxygen in solution. This was correct in so far as the evolution of air required time, but there were several boiling-out processes for determining the gases dissolved in water which gave substantially exact results, although Leduc had shown that freezing was the most efficient means of degassing water. Of course, water at $100^{\circ} \mathrm{C}$. under air, when the partial pressure of the air was $760 \mathrm{~mm}$., would dissolve about $3 \mathrm{~cm}^{3}$ per litre of oxygen. In any physical degassing process

\footnotetext{
* i.e., nitrogen plus argon.
} 
based on the use of an elevated temperature or reduced pressure or both, it was important to use appropriate means-spraying, dripping through small apertures, agitation, etc.-to obtain as large a surface of contact between the water and the atmosphere to which it was exposed.

Mr. Percy G. Jackson wrote that he did not entirely agree with the Author's remarks in connexion with the continuous blowingdown of boilers. He had known cases in which this had been particularly useful, but he did not look upon it as generally necessary. The Author stated that, in order that periodic blowing-off from the lowest point in the boiler might be effective, a large quantity of water must be blown off at each operation. In the writer's opinion this was not at all necessary. If the periodical blowing-off was for the purpose of preventing the accumulation of mud, the opening of the blow-off cock for a few seconds at a time was all that was necessary.

He deprecated most strongly the idea of returning the blown-off water to the boiler. Though the Author removed the suspended impurities by passing the blow-off water through a settling chamber, he (the writer) did not see how any of the saline impurities could be removed, as the Author appeared to suggest. No boiler should be worked with a saline concentration exceeding about 5,000 grains per gallon, and as the solubility of all salts likely to be present in the water exceeded this, even at a temperature as low as $25^{\circ} \mathrm{C}$., he could not understand how they could be removed by the simple process of cooling the blow-off water; and in the case of calcium sulphate, if present, this being more soluble at the lower temperatures than at the temperature of the boiler, it would have a tendency to redissolve rather than to be precipitated. As the prevention of undue accumulation of saline constituents was one of the two purposes for which blowing down was desirable, it appeared that the object was defeated by returning it to the boiler. The lower the saline concentration in the boiler the better, particularly if nitrates were present. The heat lost by allowing blown-off water to run to waste was so slight, provided the blowing-off was not excessive, that he could see no useful purpose in returning it to the boiler. 
Mr. Philis L. Riviere wrote that he would like to support very strongly all that.M. Kestner had said on the necessity for closer co-operation between the chemist and the engineer. At the London County Council Tramways Power Station at Greenwich, where for some considerable time now they had been fortunate in having the assistance of a fully qualified chemist working under the supervision of the London County Council Chief Chemist, the results following on the chemical work carried out had been most gratifying.

With regard to the question of blowing-down boilers, they had had at Greenwich the usual difficulties in deciding on the proper quantity of water to be blown out from time to time, and they were not assisted to any great extent by considering the procedure adopted elsewhere, as there appeared to be no standard test adopted for the determination of the degree of concentration of salts in boiler waters, and no agreed definite limit to the degree of concentration of solids allowable before blowing down was considered necessary. As a result of the work carried out by the chemist, it was decided that the chlorine content of the boiler water should be taken as a measure of the solids present in the water, and a figure of 400 parts of chlorine per 100,000 parts of boiler water was taken as representing the concentration allowable in ordinary circumstances. When boiler water was analysed and the chlorine content ascertained, the "alkalinity" was also taken, and for the conditions existing at Greenwich, it had been found that Chlorine + Alkalinity $\times 3=$ the total soiids present in parts per 100,000 .

When taking samples of water from boilers for analysis, it had been found that great care should be taken to ascertain which position on the boiler, was most likely to give samples that would be consistent and furnish reliable figures on which the general condition of the boiler water could be determined. Under the conditions prevailing at Greenwich, where Babcock and Wilcox boilers were set in pairs and were fed through feed check-valves on each drum supplied by means of a pipe controlled by a hand regulated feedvalve for each boiler, it was found that the concentration of solids in the outside drum was usually from 4 to 5 times as high as the concentration in the inside drum. The analysis of the feed-water at 
Greenwich gave the following general results over a period of three months : Alkalinity 0.91 , Chlorine 0.71 , Hardness 0.73 , while for the same period the corresponding figures for the make-up feed were: Alkalinity $6 \cdot 8$, Chlorine $2 \cdot 5$, Hardness $4 \cdot 2$, all figures representing parts per 100,000. It had been found that, in the case of the Babcock and Wilcox boilers, which were emptied and refilled with ordinary feed-water once every six or seven weeks when cleaning was carried out, the concentration of solids never exceeded 200 parts of chlorine per 100,000, and therefore no blowing down was necessary beyond opening and closing the blowdown cocks once per week to ensure that they were in working order.

In the case of the Stirling boilers, the allowable concentration had been temporarily restricted to 50 parts of chlorine per 100,000 , pending an alteration to these boilers, and it was found that this figure was reached in from three to five weeks, depending on the work. done by the individual boilers. When the concentration reached the figure given, the boilers were blown down to the extent of about 250 gallons each night for four nights, when it was found that the chlorine content had been reduced from 50 to 30 parts per 100,000 , after which the boiler would usually steam for about from 10 to 14 days before blowing down was again necessary. In consequence of the comparatively small amount of water blown down, the amount of make-up feed used was a small percentage of the total water evaporated. Under modern conditions, apart from slight condenser leakage, the make-up feed being the only way in which solids could be fed into the boilers, the importance of restricting the amount of make-up feed required was at once apparent. From the results obtained at Greenwich, where the makeup feed was from $3 \frac{1}{2}$ to 5 per cent, it would appear quite reasonable to expect that' in a large modern power-station the percentage of make-up feed should not exceed from 3 to 4 per cent of the total water evaporated. The water blown out of the boilers did not as a rule exceed about 3.5 per cent of the make-up feed, but this did not include the complete changing of water when boilers were laid off for cleaning.

The water blown out of the boilers when under steam, together 
with the water emptied out when boilers were emptied for cleaning, represented about $7 \cdot 5$ per cent of the makemup feed. In order to keep a close check on the amount of make-up feed used and the amount of water blown out of the boilers, they had a meter on the make-up feed main and a "Lea" Recorder on the blow-down pipeline, the introduction of the latter having proved particularly effective in preventing waste of water, through stokers having to lower the water level in banked boilers the feed-checks on which were leaking, and through the tendency to blow out water when the water level in working boilers had become dangerously high owing to lack of attention.

From their experience at Greenwich, it was difficult to conceive of a set of conditions which would justify the installation of $M$. Kestner's continuous blow-down system, as it would appear that the limit of concentration of solids in the boiler water would have to be considerably higher than was usually considered desirable, before the action of separating out he described could take place at the temperatures mentioned. The amount of water to be blown out in the ordinary way in a well-managed boiler plant would be so small that it would be very difficult to control the quantity within reasonable limits, as considerable trouble would be expected with regulating valves which would have to be operated under very severe conditions. He would like to know whether the Author had determined the degree to which he considered the various solids contained in boiler waters might be allowed to concentrate before it was necessary to blow down through his apparatus, and which method of determining the degree of concentration he had found most reliable under ordinary operating conditions.

With regard to M. Kestner's proposals, regarding the degassing of feed-water, it would appear that, in order to keep down the quantity of deoxidizing material used to a reasonable figure, it was necessary to arrange either for a closed circuit system or that deäeration should be carried out to a certain extent by physical means before the water passed into the Paris degasser. As a result of very careful observation and readings taken over a period of seven days, it was found that, at Greenwich with a load curve as 
shown in Fig. 20, they required a hot-well capacity of about 1,200 cubic feet to provide for the variation in the quantity of water carried in the hot-wells as reserve at different periods of the day. This hot-well capacity represented about 0.026 cubic foot per $\mathrm{kw}$. of plant installed and appeared to be necessary, principally on account of the varying water-level in the boilers, of which the feed was hand-regulated. Although no doubt the capacity required in the hot-wells could be reduced considerably by the introduction of feed-water regulators, they would still require to have a large quantity

FIG. 20.

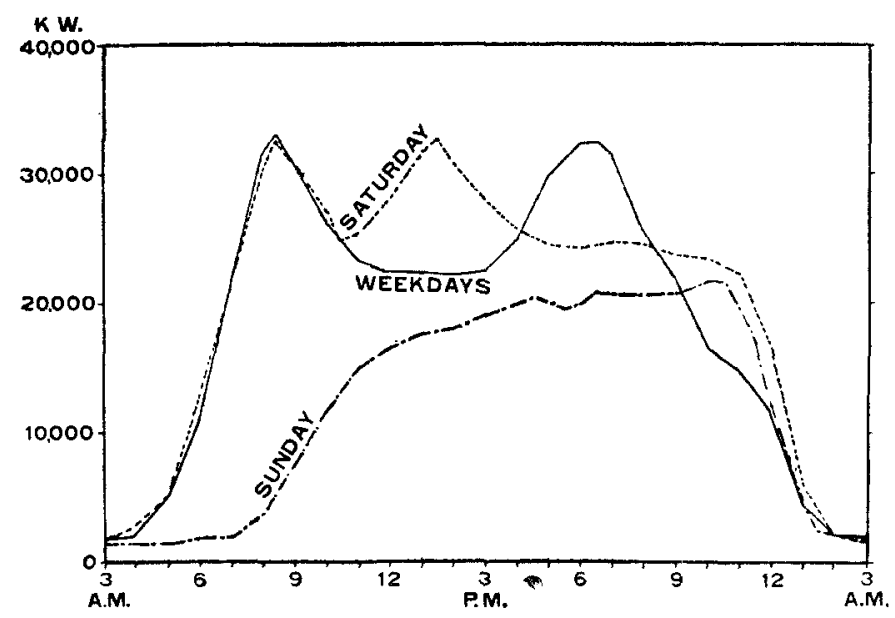

of water in reserve to provide for variation in demand on the hotwells, and it was at this point that the difficulties of degassing appeared.

When condensate left the condenser, it was, on account of the high vacuum now usually attained, almost quite free from oxygen, and in this state it was particularly liable to absorb a further quantity when exposed to the atmosphere, even for short periods. Some time ago tests were made at Greenwich to determine the amount of oxygen present in the condensate from one of the turbine sets, and 
the following figures were obtained from samples taken at the points given :-

\begin{tabular}{|c|c|c|c|c|}
\hline \multirow{2}{*}{-} & \multirow[b]{2}{*}{$\begin{array}{l}\text { Temperature } \\
\text { of Water }{ }^{\circ} \mathrm{F} \text {. }\end{array}$} & \multicolumn{3}{|c|}{ Dissolved Oxygen. } \\
\hline & & $\begin{array}{l}\text { Crm }^{3} \text { per } \\
\text { Isitre. }\end{array}$ & $\mid \begin{array}{l}\text { Lb. per } \\
\text { Million } \\
\text { Gallons. }\end{array}$ & Saturation. \\
\hline $\begin{array}{l}\text { Condensate at- } \\
\text { (1) Lea Recorder . Inlet }\end{array}$ & 70 & $0 \cdot 136$ & 1.9 & $\begin{array}{c}\text { Per cent. } \\
1 \cdot 6\end{array}$ \\
\hline (2) Lea Recorder . Outlet & 70 & $2 \cdot 14$ & $\mathbf{3} \cdot \mathbf{0}$ & $26 \cdot 2$ \\
\hline (3) Feed-Heater . Outlet & 183 & $3 \cdot 82$ & $54 \cdot 6$ & $91 \cdot 0$ \\
\hline (4) Hot-well,$\cdot$. & 110 & $4 \cdot 22$ & $60 \cdot 3$ & $93 \cdot 3$ \\
\hline
\end{tabular}

In taking tests to determine the amount of oxygen present in condensate from various sets, it had been found that, in the case of Edwards' air-pumps, dealing both with air and water, the condensate was frequently found to be super-saturated with oxygen, while, in the case of sets where the air and condensates were dealt with by separate pumps, the amount of oxygen present in the condensate was almost negligible. The difficulty of storing water free from air, and at the same time allowing for considerable variation in level, was readily apparent, and the writer would be glad if $M$. Kestner could indicate in what way he proposed to provide for suitable hot-well capacity, such as was required in large powerstations.

M. Paul Kestner wrote that modern research in the metallurgy of iron and steel had been chiefly directed to improve their physical and mechanical resistance, and it was noticeable that this had been at the expense of their chemical resistance. Steel, which replaced wrought-iron everywhere to-day, had a much lower resistance to chemical reagents and the atmosphere than the latter, and there were wrought-iron fire-bars forged in the eighteenth century which were still in a perfect state of preservation. There could be seen, in 
sugar factories in the Colonies, rails and rolling stock made at the beginning of the sugar industry which were still doing good service, whilst similar material of recent origin had rusted after a very few years' use.

Referring to Professor Hinchley's remarks (page 332), the material used in degassers was not manganese cast-iron but manganese steel, cast-iron not being readily oxidizable when exposed to the air. The presence of graphitic carbon in general indicated a feebly oxidizable substance, even in the presence of manganese. Manganese steel in which the carbon was in the combined state was most favourable for oxidation. In addition, it would be impossible to obtain very long and thin turnings if manganese cast-iron were used. There were no traces of sulphur in the turnings used.

Replying to Mr. Arthur Spyer (page 332), good results could not be obtained if ordinary turnings were employed, as they tended to pack and channels were formed. Special turnings were therefore used made in the form of ribbon from 4 feet to 5 feet long. These gave a very homogeneous mass and had proved quite successful in practice. By the old process it was found that the turnings were used up and collected in heaps ; the fillings also broke and crumbled on becoming rusty. Washing broke them still further, and the rust made them less able to absorb the oxygen. In the process of regeneration this was avoided; the mass kept its reducing properties sensibly constant until completely used up.

The distilled water produced in evaporators had the same oxygen content as the water before distillation, owing to the oxygen remaining in the vapour. This must therefore be degassed within the same limits as other boiler feed-water. If it were desired to avoid concentration in the boilers, the blow-down must be taken into account, particularly where boilers were using purified water only, without return of condensed water, as the boiler feed-water, after its passage through the purifier, contained soluble salts. These consisted of soda, sulphate of soda, and chloride of sodium. In general, carbonate of soda was present in the greatest quantity; sulphate of soda only existed to any amount in water of great permanent hardness, and the chloride of sodium only existed in water which 
contained it originally, or which contained chloride of magnesium. In all purified waters there was carbonate of lime in solution, eoncentrated to saturation point at the temperature at which purification took place. All these salts concentrated in the boiler except the carbonate of soda, which concentration was limited by the continuous blow-down, and the carbonate of calcium, whose solubility was low and could not exceed its saturation at the boiler temperature. The latter salt gave rise to the formation of muds which were removed by the continuous blow-down and deposited in the purifier ; thus there remained sulphate of soda and chloride of sodium. These salts concentrated until they were deposited in the purifier, due to the difference of solubility at the different temperatures obtaining in the boiler and purifier, these deposits being in proportion to the difference of saturation percentages at the two temperatures. This concentration represented for each of these two salts more than 300 grammes per litre. Such an increase would produce mud in the boilers and an elevation of the boiling point ; thus it must be avoided.

Mud was produced in the boilers from the moment that a certain limit of viscosity was passed; and the latter was a function of the concentration and the temperature. The elevation of boiling point followed the Raoult's law. If one exterpolated for the saturation concentrations, and applied the formula

$$
\mathrm{T}=\frac{520 \times \mathrm{C}}{\mathrm{M}}
$$

$\mathrm{C}$ being the concentration, and $\mathrm{M}$ the molecular weight of salt dissolved, then it was found for a concentration of 1 per cent an increase of 0.088 and for 30 per cent $2 \cdot 64$. The elevation of boiling point corresponded to a very slight diminution of vapour tension and did not sensibly affect evaporation in the boilers. In practice it was found impossible to obtain perfectly dry steam, and, if the concentration in the boiler were raised, the water entrained by the steam would contain a larger quantity of dissolved salts and thus a larger quantity in the condensed water. The presence of these salts in the steam was apt to deposit sediment in the tubes of the superheaters and to attack the turbine blading. The concentration 
in the boilers must therefore be limited, the maximum depending on the initial concentration of dissolved salts in the purified water, and this limit was again dependent upon the type of boiler.

It has just been shown that there are numerous physical reasons for avoiding concentration in boilers; but on the other hand, reasons of a chemical nature opposed too low concentrations. The work of Friend and Brown and Heyn and Bauer, had proved that there existed for each salt critical low concentrations for which the attack of the iron was a maximum, and that this attack diminished sensibly for higher concentrations. For carbonate of soda a concentration was rapidly reached, not only where the attack of the iron was stopped, but even where the soda protected the metal against the action of other salts; whilst the action of chloride and sulphate of soda decreased proportionately with the concentration, but at saturation point it was still appreciable. It was necessary, according to the above-mentioned Authors, that the soda should reach a concentration in the boiler of more than 1 per cent, and it could be affirmed that for concentration of 1 per cent, the corrosive action of the other salts was sensibly lessened. This latter case corresponded with that obtained with continuous blow-down. To sum up, in the case of purification by continuous blow-down, the limitation of concentration in the boilers ought to be governed by physical reasons and be determined quantitatively for each case.

There were two methods, chemical and physical, for limiting the salt content in boilers :- -

The Chemical method applied particularly to waters rich in sulphate of calcium. It consisted of introducing in the purifer carbonate of baryta which, by double decomposition with the sulphate of calcium, gave carbonate of calcium and sulphate of baryta. The two latter salts were extremely insoluble, and allowed complete elimination of the sulphate of calcium. No method was at present known for the elimination of chloride of sodium.

The Physical method was the simple one of blowing down the boilers periodically. The concentration in the boilers obviously would not increase if the quantity of salt which entered in a day was equal to that which was blown down in the same time. 
Let $\mathrm{N}$ be the number of cubic metres of raw water introduced in the boiler in twenty-four hours.

$c$ the concentration of the raw water in grammes of salts per cubic metre.

$\mathrm{V}$ the volume of the boiler in cubic metres.

C the concentration limit of the salts in the boiler in grammes per cubic metre.

$\frac{1}{p}$ the fraction of the volume of the boiler emptied by the purge in twenty-four hours.

The quantity of salts entering the boiler for twenty-four hours is $\mathrm{Nc}$.

The quantity of salts eliminated in twenty-four hours is

$$
\mathrm{rC} \times \frac{1}{p}
$$

Ijet these quantities be equated then

$$
\mathrm{Nr}=\mathrm{CV} \frac{1}{p} \text {. }
$$

The concentration $\mathrm{C}$ found in the boiler at equilibriums will be

$$
\mathrm{C}=\frac{\mathrm{N} c p}{\mathrm{~V}} \text {. }
$$

Note that $\frac{N}{\bar{V}}$ is the number of boilers filled per day with the raw water.

Let $\quad \mathrm{A}=\frac{\mathrm{N}}{\mathrm{i}}$,

then

$$
\mathrm{C}=c \times \mathbf{A} \times p .
$$

The final concentration of the boiler would then be equal to the product of the concentration of the raw water by the number of boilers of raw water evaporated per twenty-four hours and by the number of blow-downs necessary to empty the contents of one boiler. In other words, it was proportionate to the concentration of the raw water by the output of the boiler, and inversely to the quantity of the blow-down.

The preceding calculation enabled the following curve to be drawn, Fig. 21. It was thus seen that the concentrations might be limited without important losses of hot water, and blowing down 
FIG. 21.-Concentration Limit of Water from Boilers for the Watcr Circulation containing 0.1 Gramme per Litre, of Dissolved Salt.

Concentration in Grammes per Litre.

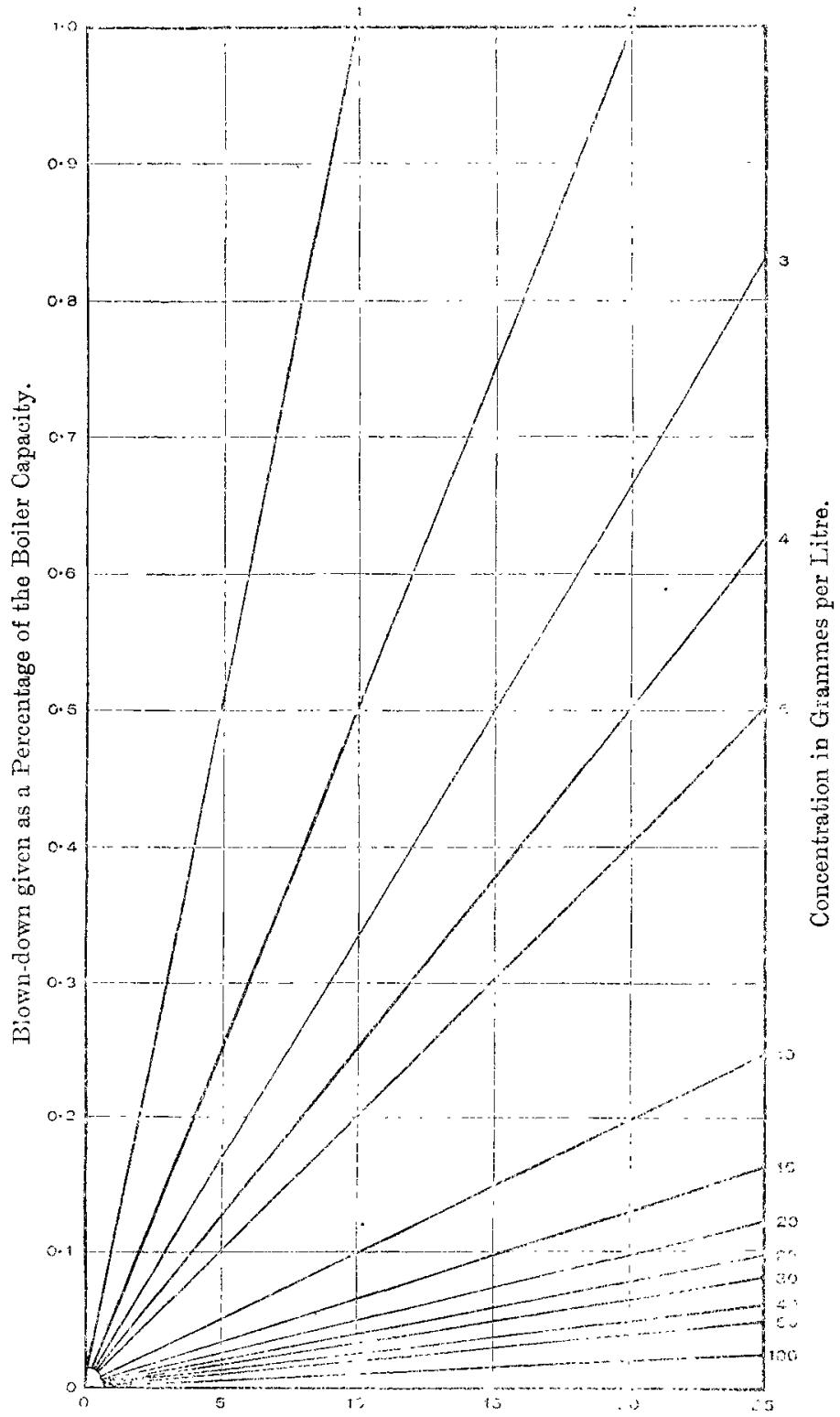

No. of Boilers of Raw Water Fvaporated Daily. 
was not so important a factor where the salt content in the water was low. The installations most unfavourable were those which evaporated purified water and which did not recover the condensed steam. In those cases where the condensed steam was reintroduced into the boiler, it was only necessary to blow down a small quantity. Boilers using distilled water similarly only blew down a very small percentage, since the distilled water contained a very small quantity of salts. It had been found that continuous blow-down was the least costly method of purification. As much on account of the price of the plant as of their running cost. Added to these advantages was the fact that no incrustations were formed in the boiler and that consequently cleaning was unnecessary. Galvanic action did not appear to play any large part in the corrosion of boilers.

In reply to $\mathrm{Mr}$. R. W. Bailey (page 334) the Author stated that the employment of the special ribbon gave excellent results, and did not allow of short circuits or channelling (see reply to $\mathrm{Mr}$. Spyer). The average relationship established between the speed of degassing at low and high temperatures was shown on Fig. 13 (page 320). So many factors intervened that it was impossible to obtain a general formula, and relationships could only be established for particular cases. Tests had been made on expanded metal, but the net cost was about three times that of iron turnings for the same output, and for the same weight the turnings had a surface about four times greater.

With reference to Professor Donnan's remarlss (page 338), chemical degassing was inapplicable to drinking water; the corrosion in town's water pipes must be overcome, either by the action of an incrusting coating by a lining of cement in the iron piping, or by using armoured cement feeders.

In reply to Professor Brame, the Author stated that what took place in a boiler at $200^{\circ} \mathrm{C}$. could not be observed; but when it was opened, rust was found as a result of the attack of the iron. It was probable that others had ascertained the transformation of the oxides of iron. Without referring to Gmelin or to Hermes, references would be found in the most recent works and more on a level with actual experience (see Bibliography, page 360-article of Von Siegmon). All possible products between $\mathrm{FeO}$ and $\mathrm{Fe}_{2} \mathrm{O}_{3}$ and their 
hydrates were produced in this blackish-green oxide. The product that had been examined had a percentage of oxygen which did not correspond either to one of the oxides or one of the hydrates. In other cases, different results had been obtained. It seemed that the temperature played a great part in the degree of oxidation of the final product. At the present moment researches had not been finished on the subject of this state of oxidation of the iron.

With regard to the subject of chloride of magnesium (page 340), it had not been found that a small amount of soda in the water modified the action. It was thought this was due to the very great oxidizable properties of the iron turnings.

In reply to Mr. Bailey's communication, irregularity or "spottiness" had never been observed in the layer of oxide in degassers where iron turnings were used; it always had a very uniform colour. Degassing by atomizing the water in a space containing steam could only give good results by aspirating with a pump the gases and steam which were in contact with the water. This was done in the Elliott process by the use of a high vacuum and vaporization of the water which had, under this small pressure, an almost explosive transformation into steam. The extraction of the steam necessitated a powerful pump; the condensed steam contained air which had to be eliminated. If it were sent again through the plant, a continual enrichment in air was produced in the water to be degassed. There was no doubt that good results had been obtained by this process, but it was open to the following objections:-

(1) It was difficult to obtain sufficient vacuum when the amount of water to be degassed was large.

(2) In this plant, where the water and the steam were under a very reduced pressure, particularly so in the water extraction pump, a very small leak sufficed to destroy the effect of the degassing.

In regard to the communication by Messrs. Coste and Powney, the facts quoted on the subject of sulphate of calcium were known; that was why in the text, it was stated that the diminution of solubility of the $\mathrm{CaSO}_{4}$ produced by the elevation of temperature was of very little importance in that case. The reference quoted on the subject of $\mathrm{MgCl}_{\text {? }}$ was not known, but there had been no 
cases observed of corrosion by chloride of magnesium where the water was alkaline; but there were numerous examples in the case of collieries where the water was neutral, or slightly acid. On the other hand, it was not contended that purification by soda alone was better than by lime and soda; the point being that, in the particular case of continuous blow-down, purification by soda was more advantageous.

As to the condensed water regaining oxygen, it was very difficult to avoid in an ordinary or even specially made circuit; pumps and hot-wells of variable level were also causes of air re-entering. The question was solved in the following way :-

(1) Maintain a small pressure in the total water circuit, thus leaks could only take place from the interior to the exterior. All pumps should be under load as much as possible, thus there could be no re-entering of air.

(2) The degasser was under load and placed immediately in front of the feed-pump.

(3) For hot-wells of variable level, in the circuit type there was always feed-water at high temperature, thus a cushion of steam could be maintained over this water to create pressure in the space of hot-well not filled by water and to a void re-entering of air. The special hot-wells were combined with a degassing device placed therein, which removed the oxygen from the water before it left the hot-well; thus there could be large variations in the demand for water.

As for elimination of gases from the water by boiling, it must be remarked that this only took place completely by prolonged boiling, and that it would be impossible industrially to degass by such boiling which would necessitate removal of an important quantity of water at the same time as the gas. An equilibrium was established between the partial pressure of the oxygen in the steam of the oxygen content of the water; in order that this should be nil, the steam must be pure. It must be noted that the oxygen content of the water was infinitely small in comparison with the volume of steam that this water represented.

Continuous blow-down was the cheapest process, and if it were 
a question of particularly difficult cases, as Mr. Jackson indicated, it was generally advisable to apply it throughout. Continuous blowdown had the one aim of avoiding calcareous precipitation in the boilers. It was not a question of attaining concentrations, or sulphate of soda, for example, would be precipitated, but only of limiting the concentration of carbonate of soda and producing precipitation in the purifier by difference in temperature of part of the $\mathrm{CaCO}_{3}$ of the blow-down.

With regard to Mr. Riviere's remarks, the question of purification ought not to be considered only from the point of view of central stations where 98 per cent of condensed water was recovered, but from that of the more numerous works using purified water only, which did not recover their condensed water or use it for other purposes than feeding the boilers.

\section{Bibliography.}

Die Bekampfung von Rost and Abzehrungen an Dampfkesseln, by E. Hohn. Zurich 1919.

Die Ursachen der Zerstorungen an Wasserrohrkesseln im Turbinenbetrieb und die zu ergreifenden Gegenmassnahmen, by Von Seigmon, Zeits. der Ver. deut. Ing. Nos. 21, 22, 23 May 1919.

Dampfkessel schäden and Ihre Möglichste Veihutung, by Frantz. Kattowitz, 1915.

Die Reinigung des Kesselspeisewassers, by Claussen. Leipzig, 1921.

Epuration perfectionné des eaux pour Chaudiers. Paris, Chemic et Industrie, Dec. 1920.

Les filtres à tournure de fer et leur emploi pour la suppression des corrosions. Paris, Chaleur et Industrie, July 1920.

Enquête sur les grandes centrales thermiques, by H. Dieterlen, Chaleur et Industrie, Nos. 1 and 2, 1920.

The Corrosion of Iron and Steel, by Newton Friend, Longmans Green, 1911.

Zeitgemasse Kesselanlagen fur Elekhesehe Kraftwerke, by Ott. Hanover, 1920-21. 